\title{
On strong integrability of the dressing cosets
}

\author{
Ctirad Klimčík \\ Aix Marseille Université, CNRS, Centrale Marseille \\ I2M, UMR 7373 \\ 13453 Marseille, France
}

\begin{abstract}
We formulate sufficient conditions for the strong integrability of dressing cosets. We provide several sigma-model backgrounds solving those conditions, some of them are new and some of them were not so far formulated as the dressing cosets. The new models are based on the Drinfeld doubles having the structure of higher order jet bundles of quadratic Lie groups.
\end{abstract}

\section{Introduction}

The dressing cosets are particular nonlinear $\sigma$-models in two dimensions which were originally introduced in [35] in a successfull attempt to generalize the socalled Poisson-Lie T-duality [33]. The first order Hamiltonian dynamics of the dressing cosets can be described in terms of the dynamical systems referred to as degenerate $\hat{\mathcal{E}}$-models which themselves generalize the so called non-degenerate $\mathcal{E}$-models. This generalization is substantial, nevertheless it received a relatively little attention in the literature so far although there was some older activity in the field $[5,6,20,21,46,48,49]$, just like a more recent one $[13,14,18,30,32,43,45]$.

We believe that the dressing cosets as well as the degenerate $\hat{\mathcal{E}}$-models are going to play an increasing role in future in all those places where their simpler non-degenerate counterparts already play role, like in the theory of integrable $\sigma$ models $[1,2,7,10,12,15,16,23-26,41,42,47]$. In particular, it is well-known that many integrable $\sigma$-models on group manifolds can be in fact interpreted in terms of the non-degenerate $\mathcal{E}$-models $[19,27-29,31,37]$. However, in the case of the integrable models living on the coset manifold an unified interpretation in terms of the dressing cosets is so far missing. Among other things, we fill this gap at least partially in the present paper.

It is important to distinguish between a weak and a strong integrability of a non-linear $\sigma$-model. The weak integrability means that the equation of motion of 
the model can be written in the so called Lax form with spectral parameter $z$, that is in the form

$$
\frac{d L(z)}{d \tau}=[L(z), M(z)]
$$

where $L(z)$ and $M(z)$ are $z$-families of matrix-valued functions on the phase space of the $\sigma$-model. The strong integrability then means that every two spectral invariants of the Lax matrix $L(z)$ Poisson commute, for whatever values of the spectral parameters $z$ and $w$. As we shall see later on, this means that the matrix Poisson bracket of the Lax matrix $L(z)$ with $L(w)$ is governed by the so called $r$-matrix $r(z, w)$, which is an important characteristics of the strongly integrable model.

Sufficient conditions for the weak integrability of the non-degenerate $\mathcal{E}$-models were first formulated in [44] and solved in many cases in [37]. They were later extended in [32] to ensure also the strong integrability. In this paper, we formulate the sufficient conditions for the strong integrability of the degenerate $\hat{\mathcal{E}}$-models and we solve them for several classes of the dressing cosets. Some of those classes constitute a reinterpretation of the known symmetric spaces $\sigma$-models in terms of the dressing cosets while the others are completely new and are based on high order jet bundles of quadratic Lie groups interpreted as the Drinfeld doubles. In particular, the jet bundle $J^{2 n+1} G$ of a compact semi-simple Lie group $G$ gives rise to the strongly integrable dressing coset describing a suitable interaction of $n$ fields with values in the Lie algebra $\mathcal{G}$ of the group $G$.

The plan of the paper is as follows: In Section 2, we review the concept of the non-degenerate $\mathcal{E}$-model as well as the sufficient conditions of its integrability (the weak as well as the strong ones). In Section 3.1, we review in much technical detail the concepts of the degenerate $\hat{\mathcal{E}}$-models as well as that of the dressing cosets while in Section 3.2 we start with the presentation of the original results, namely, we formulate the sufficient conditions for the strong integrability of the dressing cosets. In Section 4, we interpret a non-deformed, a $\lambda$-deformed and an $\eta$-deformed symmetric space $\sigma$-models as the dressing cosets and we show that the well-known strong integrability of those three theories can be understood in an unified way within our degenerate $\hat{\mathcal{E}}$-model formalism. In Section 5 , we solve our sufficient conditions of the strong integrability for a new family of the dressing cosets which is parametrized by positive integers. Thus, for given $n$, the model describes a suitable integrable interaction of $n \mathcal{G}$-valued fields. Only for $n=1$ the resulting $\sigma$-model was known previously, it is in fact the pseudo-chiral model of Zakharov and Mikhailov [53]. We determine also the so called twist function of the generalized pseudo-chiral integrable model for every $n$. In Section 6 , we provide conclusions and an outlook. 


\section{Reminder: non-degenerate $\mathcal{E}$-models}

\subsection{The first and the second order formulations}

In this paper, by a Drinfeld double $D$ we shall understand a connected evendimensional Lie group equipped with a bi-invariant pseudo-Riemannian metric of maximally-Lorentzian (split) signature. An $\mathcal{E}$-model, introduced in [27,33,34], is a first-order Hamiltonian dynamical system $\left(\omega, H_{\mathcal{E}}\right)$ living on the loop group $L D$ of the Drinfeld double. The symplectic form $\omega$ and the Hamiltonian $H_{\mathcal{E}}$ on $L D$ are respectively given by the formulas

$$
\begin{gathered}
\omega=-\frac{1}{2} \oint\left(l^{-1} \delta l \wedge\left(l^{-1} \delta l\right)^{\prime}\right)_{\mathcal{D}} \\
H_{\mathcal{E}}=\frac{1}{2} \oint\left(l^{\prime} l^{-1}, \mathcal{E} l^{\prime} l^{-1}\right)_{\mathcal{D}} .
\end{gathered}
$$

Here $(., .)_{\mathcal{D}}$ is the non-degenerate ad-invariant symmetric bilinear form $(., .)_{\mathcal{D}}$ defined on the Lie algebra $\mathcal{D}$ of the Drinfeld double (it is given by the pseudoRiemannian metric at the group origin). The integration in Eqs. (2.1), (2.2) is over the loop parameter $\sigma$, while the derivative with respect to the loop parameter is denoted by the apostrophe. The symbol $\delta$ stands for the de Rham exterior differential on the loop group $L D$, the closed string configuration $l$ parametrizes $L D$ and, finally, $\mathcal{E}: \mathcal{D} \rightarrow \mathcal{D}$ is an $\mathbb{R}$-linear operator squaring to identity, symmetric with respect to the bilinear form $(., .)_{\mathcal{D}}$ and such that the bilinear form $(., \mathcal{E} \text {. })_{\mathcal{D}}$ on $\mathcal{D}$ is strictly positive definite.

It turns out, that the left action of the group $L D$ on itself is generated by the moment map $j=l^{\prime} l^{-1}$. The components of the current $j$ then verify the Poisson current algebra

$$
\left\{\left(j\left(\sigma_{1}\right), T_{1}\right)_{\mathcal{D}},\left(j\left(\sigma_{1}\right), T_{2}\right)_{\mathcal{D}}\right\}=\left(j\left(\sigma_{1}\right),\left[T_{1}, T_{2}\right]\right)_{\mathcal{D}} \delta\left(\sigma_{1}-\sigma_{2}\right)+\left(T_{1}, T_{2}\right)_{\mathcal{D}} \delta^{\prime}\left(\sigma_{1}-\sigma_{2}\right)
$$

which plays a crucial role in the dynamics of the $\mathcal{E}$-models. Indeed, the Poisson brackets (2.3) as well as the explicit form (2.2) of the Hamiltonian give the following first order equations of motion of the $\mathcal{E}$-model

$$
\frac{\partial j}{\partial \tau}=\left\{j, H_{\mathcal{E}}\right\}=(\mathcal{E} j)^{\prime}+[\mathcal{E} j, j]
$$

Consider the $\mathcal{E}$-model on the Drinfeld double $D$ and let $K \subset D$ be a halfdimensional subgroup such that the Lie subalgebra $\mathcal{K}$ is isotropic with respect to the bilinear form $(., .)_{\mathcal{D}}$. Then it was shown in [36] that there is a two-dimensional non-linear $\sigma$-model such that its first order dynamics can be expressed in terms of the $\mathcal{E}$-model; in particular its first order Hamiltonian equations of motion are given by (2.4). The action of this $\sigma$-model reads

$$
S_{\mathcal{E}}(l)=\frac{1}{4} \int \delta^{-1} \oint\left(\delta l l^{-1},\left[\partial_{\sigma} l l^{-1}, \delta l l^{-1}\right]_{\mathcal{D}}\right)_{\mathcal{D}}+
$$




$$
+\frac{1}{4} \int d \tau \oint\left(W_{l}^{+} \partial_{+} l l^{-1}, \partial_{-} l l^{-1}\right)_{\mathcal{D}}-\frac{1}{4} \int d \tau \oint\left(\partial_{+} l l^{-1}, W_{l}^{-} \partial_{-} l l^{-1}\right)_{\mathcal{D}} .
$$

Here $l(\tau, \sigma) \in D$ is a field configuration, $\partial_{ \pm}=\partial_{\tau} \pm \partial_{\sigma}, \delta^{-1}$ is a (symbolic) inverse of the de Rham differential and $W_{l}^{ \pm}: \mathcal{D} \rightarrow \mathcal{D}$ are the projectors fully characterized by their respective kernels and images

$$
\operatorname{Ker}\left(W_{l}^{ \pm}\right)=A d_{l}(\mathcal{K}), \quad \operatorname{Im}\left(W_{l}^{ \pm}\right)=(1 \pm \mathcal{E}) \mathcal{D} .
$$

It may seem, that the $\sigma$-model (2.5) lives on the target $D$ but, actually, it lives on the space of cosets $D / K$ because it enjoys the gauge symmetry

$$
l(\tau, \sigma) \rightarrow l(\tau, \sigma) k(\tau, \sigma), \quad k(\tau, \sigma) \in K .
$$

In some cases, the fibration $D \rightarrow D / K$ admits a global section $p: D / K \rightarrow D$, in which case we can fix the gauge $l=p$ in the action (2.5).

Note also that the equations of motion of the $\sigma$-model (2.5) can be written as

$$
\partial_{+}\left(W_{l}^{-} \partial_{-} l l^{-1}\right)-\partial_{-}\left(W_{l}^{+} \partial_{+} l l^{-1}\right)-\left[W_{l}^{+} \partial_{+} l l^{-1}, W_{l}^{-} \partial_{-} l l^{-1}\right]_{\mathcal{D}}=0 .
$$

\section{$2.2 \quad$ Integrability of the non-degenerate $\mathcal{E}$-models}

Suppose that there is the $\mathcal{E}$-model based on the Drinfeld double $D$ and there is also a quadratic Lie algebra $\mathcal{G}$ together with an one-parametric family of linear operators $O(z): \mathcal{D} \rightarrow \mathcal{G}$ verifying the following conditions

$$
\left[O(z) x_{+}, O(z) x_{-}\right]_{\mathcal{G}}=O(z)\left[x_{+}, x_{-}\right]_{\mathcal{D}}, \quad x_{ \pm} \in \mathcal{D}, \quad \mathcal{E} x_{ \pm}= \pm x_{ \pm} .
$$

It was shown in $[37,44]$ that Eq. (2.9) is the sufficient condition for the existence of the Lax pair $L(z), M(z)$ of this $\mathcal{E}$-model. The operators $L(z), M(z)$ act on the loop Lie algebra $L \mathcal{G}$ and they are given by the formulas

$$
\begin{gathered}
L(z)=\partial_{\sigma}-\operatorname{ad}_{O(z) j}^{\mathcal{G}}, \\
M(z)=-\operatorname{ad}_{O(z) \mathcal{E} j}^{\mathcal{G}} .
\end{gathered}
$$

The condition (2.9) guarantees that the field equations (2.4) of the $\mathcal{E}$-model can be represented in the Lax form with spectral parameter [38]

$$
\left\{L(z), H_{\mathcal{E}}\right\}=\frac{d L(z)}{d t}=[L(z), M(z)] .
$$

The Lax form (2.12) of the field equations guarantees the so called weak Lax integrability, which means that the spectral invariants (typically the traces of the powers) of the operator $L(z)$ are the integrals of motion. If, however, those integrals of motions Poisson commute among each other, we say that the $\mathcal{E}$-model is strongly Lax integrable $[4,40,50]$. 
The sufficient conditions for the strong Lax integrability of $\mathcal{E}$-models were formulated in [32]. It is thus required that it exists a non-degenerate invariant symmetric bilinear form $(., .)_{\mathcal{G}}$ on $\mathcal{G}$ and there is a two-parametric family of operators $\hat{r}(z, w): \mathcal{G} \rightarrow \mathcal{G}$ such that it holds

$$
\begin{gathered}
{\left[O^{\dagger}(z) x, O^{\dagger}(w) y\right]_{\mathcal{D}}+O^{\dagger}(z)[x, \hat{r}(z, w) y]_{\mathcal{G}}+O^{\dagger}(w)[\hat{r}(w, z) x, y]_{\mathcal{G}}=0, \quad \forall x, y \in \mathcal{G},} \\
\left(O^{\dagger}(z) x, O^{\dagger}(w) y\right)_{\mathcal{D}}+(x, \hat{r}(z, w) y)_{\mathcal{G}}+(\hat{r}(w, z) x, y)_{\mathcal{G}}=0, \quad \forall x, y \in \mathcal{G} .
\end{gathered}
$$

Here $O(z)^{\dagger}: \mathcal{G} \rightarrow \mathcal{D}$ is the adjoint of the operator $O(z)$; it is defined by the relation

$$
(O(z) x, y)_{\mathcal{G}}=\left(x, O^{\dagger}(z) y\right)_{\mathcal{D}}, \quad \forall x \in \mathcal{D}, y \in \mathcal{G} .
$$

To see, why (2.13) and (2.14) are the sufficient conditions of the strong Lax integrability, we use them as well as Eq.(2.3) to calculate the Poisson brackets of the matrix elements of the Lax operator $L(z)$

$$
\begin{gathered}
\left\{\left(y^{\prime}, L(z)\left(\sigma_{1}\right) x^{\prime}\right)_{\mathcal{G}},\left(y^{\prime \prime}, L(w)\left(\sigma_{2}\right) x^{\prime \prime}\right)_{\mathcal{G}}\right\}=\left\{\left(O(z) j\left(\sigma_{1}\right), x\right)_{\mathcal{G}},\left(O(w) j\left(\sigma_{2}\right), y\right)_{\mathcal{G}}\right\}= \\
=\left(j\left(\sigma_{1}\right),\left[O^{\dagger}(z) x, O^{\dagger}(w) y\right]_{\mathcal{D}}\right)_{\mathcal{D}} \delta\left(\sigma_{1}-\sigma_{2}\right)+\left(O^{\dagger}(z) x, O^{\dagger}(w) y\right)_{\mathcal{D}} \partial_{\sigma_{1}} \delta\left(\sigma_{1}-\sigma_{2}\right)= \\
=-\left(\left(O(z) j\left(\sigma_{1}\right),[x, \hat{r}(z, w) y]_{\mathcal{G}}\right)_{\mathcal{G}}+\left(O(w) j\left(\sigma_{2}\right),[\hat{r}(w, z) x, y]_{\mathcal{G}}\right)_{\mathcal{G}}\right) \delta\left(\sigma_{1}-\sigma_{2}\right) \\
-\left((x, \hat{r}(z, w) y)_{\mathcal{G}}+(\hat{r}(w, z) x, y)_{\mathcal{G}}\right) \partial_{\sigma_{1}} \delta\left(\sigma_{1}-\sigma_{2}\right) .
\end{gathered}
$$

where we have set

$$
x=\left[y^{\prime}, x^{\prime}\right]_{\mathcal{G}}, \quad y=\left[y^{\prime \prime}, x^{\prime \prime}\right]_{\mathcal{G}}, \quad x^{\prime}, x^{\prime \prime}, y^{\prime}, y^{\prime \prime} \in \mathcal{G} .
$$

The relation (2.16) can be rewritten in the operator form as

$$
\{L(z) \otimes \mathrm{Id}, \mathrm{Id} \otimes L(w)\}=[r(z, w), L(z) \otimes \mathrm{Id}]-\left[r^{p}(w, z), \mathrm{Id} \otimes L(w)\right],
$$

where the operator $r(z, w)$ acts on the tensor product $L \mathcal{G} \otimes L \mathcal{G}$ and it is defined as

$$
r(z, w)=C_{A B} \operatorname{ad}_{\hat{r}(z, w) T^{A}} \otimes \operatorname{ad}_{T^{B}} \delta\left(\sigma_{1}-\sigma_{2}\right) .
$$

Note that $C_{A B}$ is the inverse matrix of $C^{A B}$ defined by

$$
C^{A B}:=\left(T^{A}, T^{B}\right)_{\mathcal{G}}
$$

where $T^{A}$ is some basis of the Lie algebra $\mathcal{G}$ on the choice of which actually the $r$-matrix (2.19) does not depend. The notation $r^{p}$ means

$$
r^{p}=\sum_{\alpha} B_{\alpha} \otimes A_{\alpha}
$$

if $r$ has the form

$$
r=\sum_{\alpha} A_{\alpha} \otimes B_{\alpha}
$$


for some family of linear operators $A_{\alpha}, B_{\alpha}$ acting on $L \mathcal{G}$. The spectral parameter $z$ may even take complex values in which case the crucial map $O(z)$ is considered as the map from $\mathcal{D}$ to $\mathcal{G}^{\mathbb{C}}$.

By the general theory of the Lax integrable system exposed e.g. in the book [3], the validity of the identity (2.18) precisely guarantees the strong integrability of the dynamical system possessing the Lax operator $L(z)$.

\section{Degenerate $\hat{\mathcal{E}}$-models}

\subsection{Reminder: dressing cosets}

Consider again the $\mathbb{R}$-linear involution $\mathcal{E}: \mathcal{D} \rightarrow \mathcal{D}$ symmetric with respect to the bilinear form $(.,)_{\mathcal{D}}$ and such that the bilinear form $(., \mathcal{E} \text {. })_{\mathcal{D}}$ on $\mathcal{D}$ is strictly positive definite. Furthermore, we suppose

1) There is an isotropic subgroup $F \subset D$ such that the involution $\mathcal{E}$ commutes with the adjoint action of $F$ on $\mathcal{D}$;

2) The restriction of the bilinear form $(., \mathcal{E} \text {. })_{\mathcal{D}}$ to the Lie subalgebra $\mathcal{F}$ is strictly positive definite.

In [30], the so called degenerate $\hat{\mathcal{E}}$-model was associated to the data just described as a slight reformulation of the so called dressing coset introduced in [34] (in what follows, we use the both terms interchangeably). Thus the degenerate $\hat{\mathcal{E}}$ model is the first-order Hamiltonian dynamical system obtained by an appropriate symplectic reduction of the dynamical system $\left(\omega, H_{\hat{\mathcal{E}}}\right)$, where the symplectic form $\omega$ is the standard Kirillov-Drinfeld one

$$
\omega=-\frac{1}{2} \oint\left(l^{-1} \delta l \wedge\left(l^{-1} \delta l\right)^{\prime}\right)_{\mathcal{D}}
$$

while the Hamiltonian is given by

$$
H_{\hat{\mathcal{E}}}(l)=\frac{1}{2} \oint(j, \hat{\mathcal{E}} j)_{\mathcal{D}}, \quad j:=l^{\prime} l^{-1} .
$$

Note that the dynamical system $\left(\omega, H_{\hat{\mathcal{E}}}\right)$ that we are going to reduce symplectically is not the non-degenerate $\mathcal{E}$-model in the sense of the definition given in Section 2 , inspite of the fact that the symplectic form (3.1) is the same as (2.1). This is because the linear operator $\hat{\mathcal{E}}$ appearing in the Hamiltonian (3.2) is not the same thing as $\mathcal{E}$ although it is obtained from $\mathcal{E}$ in an appropriate way. Actually, $\hat{\mathcal{E}}$ has a nontrivial kernel and, therefore, it cannot be involutive. To define $\hat{\mathcal{E}}$, we first notice that the condition 2) above means that the intersection of the Lie subalgebra $\mathcal{F} \subset \mathcal{D}$ with the linear space $\mathcal{E} \mathcal{F}$ is trivial (it contains just $0 \in \mathcal{D}$ ), which means that the Drinfeld double Lie algebra $\mathcal{D}$ can be represented as the direct sum of four $\mathrm{Ad}_{F}$-invariant vector spaces as follows

$$
\mathcal{D}=V_{+} \oplus V_{-} \oplus \mathcal{F} \oplus \mathcal{E} \mathcal{F}
$$


where the subspaces $V_{ \pm}$are defined by the conditions

$$
V_{+} \oplus V_{-}=(\mathcal{F} \oplus \mathcal{E} \mathcal{F})^{\perp}, \quad \mathcal{E} x_{ \pm}= \pm x_{ \pm}, \quad x_{ \pm} \in V_{ \pm}
$$

Note that the notation $\perp$ means the perpendicularity with respect to the bilinear form $(., .)_{\mathcal{D}}$.

Following the decomposition (3.3), every element $y \in \mathcal{D}$ can be unambiguosly written as

$$
y=y_{+}+y_{-}+y^{\mathcal{F}}+y^{\mathcal{E F}},
$$

and we can now define the operator $\hat{\mathcal{E}}: \mathcal{D} \rightarrow \mathcal{D}$ by the formula

$$
\hat{\mathcal{E}} y:=\mathcal{E}\left(y_{+}+y_{-}+y^{\mathcal{E F}}\right) .
$$

Occasionally, we shall also use the notation

$$
y^{\perp}:=y_{+}+y_{-},
$$

in particular we write

$$
y=y^{\perp}+y^{\mathcal{F}}+y^{\mathcal{E F}}
$$

and

$$
\hat{\mathcal{E}} y=\mathcal{E}\left(y^{\perp}+y^{\mathcal{E F}}\right) .
$$

It turns out that the dynamical system $\left(\omega, H_{\hat{\mathcal{E}}}\right)$ can be indeed symplectically reduced with respect to the left action of the loop group $L F$ on the loop group $L D$. To see it, we have to prove that both the symplectic form (3.1) and the Hamiltonian (3.2) are invariant with respect to the action of $L F$ on the unreduced phase space $L D$.

First we show the $L F$-invariance of the Hamiltonian $H_{\hat{\mathcal{E}}}$. Let $f \in L F$. Then we have

$$
\begin{gathered}
H_{\hat{\mathcal{E}}}(f l)=\frac{1}{2} \oint\left((f l)^{\prime}(f l)^{-1}, \hat{\mathcal{E}}(f l)^{\prime}(f l)^{-1}\right)_{\mathcal{D}}= \\
=\frac{1}{2} \oint\left(f^{\prime} f^{-1}+\operatorname{Ad}_{f}\left(\left(l^{\prime} l^{-1}\right)^{\mathcal{F}}+\left(l^{\prime} l^{-1}\right)^{\perp}+\left(l^{\prime} l^{-1}\right)^{\mathcal{E F}}\right), \mathcal{E} \operatorname{Ad}_{f}\left(\left(l^{\prime} l^{-1}\right)^{\perp}+\left(l^{\prime} l^{-1}\right)^{\mathcal{E F}}\right)\right)_{\mathcal{D}}= \\
=\frac{1}{2} \oint\left(\left(l^{\prime} l^{-1}\right)^{\mathcal{F}}+\left(l^{\prime} l^{-1}\right)^{\perp}+\left(l^{\prime} l^{-1}\right)^{\mathcal{E F}}, \mathcal{E}\left(\left(l^{\prime} l^{-1}\right)^{\perp}+\left(l^{\prime} l^{-1}\right)^{\mathcal{E F}}\right)\right)_{\mathcal{D}}=H_{\hat{\mathcal{E}}}(l)
\end{gathered}
$$

Now we show the $L F$-invariance of the symplectic form $\omega$. Let $\phi(\sigma) \in L \mathcal{F}$ and consider the vector field $v_{\phi}$ acting an a function $\Psi$ on $L D$ as

$$
\left(v_{\phi} \Psi\right)(l):=\left(\frac{d}{d s}\right)_{s=0} \Psi\left(e^{s \phi} l\right) .
$$

Since $v_{\phi}$ is the right-invariant vector field on the loop group $L D$, its contraction with the right-invariant Maurer-Cartan form $\delta l l^{-1}$ on $L D$ is

$$
\left\langle\iota_{v_{\phi}}, \delta l l^{-1}\right\rangle=\phi
$$


Then we deduce from Eqs. (3.1) and (3.12)

$$
\iota_{v_{\phi}} \omega=\delta\left(l^{\prime} l^{-1}, \phi\right)_{\mathcal{D}}=\delta(j, \phi)_{\mathcal{D}}=\delta\left(j^{\mathcal{E F}}, \phi\right)_{\mathcal{D}}
$$

which means that the quantity $-\left(j^{\mathcal{E F}}, \phi\right)_{\mathcal{D}}$ is the moment map for the left action of the element $\phi$ on $L D$.

Since the form $\omega$ is closed, its Lie derivative with respect to the vector field $v_{\phi}$ vanishes. Indeed, we have

$$
\mathcal{L}_{v_{\phi}} \omega=\left(\delta \iota_{v_{\phi}}+\iota_{v_{\phi}} \delta\right) \omega=\delta\left(\delta\left(j^{\mathcal{E F}}, \phi\right)_{\mathcal{D}}\right)=0
$$

Now we can perform the symplectic reduction of the dynamical system $\left(\omega, H_{\hat{\mathcal{E}}}\right)$ by setting

$$
j^{\mathcal{E F}}=0
$$

The reduced dynamical system is denoted as $\left(\hat{\omega}, \hat{H}_{\hat{\mathcal{E}}}\right)$ and it is referred to as the degenerate $\hat{\mathcal{E}}$-model. The reduced phase space $\hat{P}$ can be identified with the space of the left cosets $L F \backslash L D^{c}$, where $L D^{c}$ is the space of all elements of the loop group $L D$ verifying the $L F$-invariant constraint (3.15). The reduced Hamiltonian $\hat{H}_{\hat{\mathcal{E}}}$ is the restriction of the unreduced Hamiltonian $H_{\hat{\mathcal{E}}}$ from the space $L F \backslash L D$ to the space $L F \backslash L D^{c}$.

Remark 3.1. Note that even if the involution $\mathcal{E}$ satisfies the conditions 1) and 2) above, the Hamiltonian (2.2) of the non-degenerate $\mathcal{E}$-model cannot be reduced because it is not invariant with respect to the left action of the loop group $L F$ on the loop group $L D$. For this reason, the degenerate $\hat{\mathcal{E}}$-model cannot be interpreted as the symplectic reduction of the non-degenerate one.

The equations of motion of the unreduced dynamical system $\left(\omega, H_{\hat{\mathcal{E}}}\right)$ obviously read

$$
\frac{\partial j}{d \tau}=\left\{j, H_{\hat{\mathcal{E}}}\right\}=(\hat{\mathcal{E}} j)^{\prime}+[\hat{\mathcal{E}} j, j]_{\mathcal{D}}
$$

or, in the decomposed form following the decomposition (3.3), as

$$
\begin{aligned}
\frac{\partial j_{+}}{\partial \tau} & =j_{+}^{\prime}+2\left[j_{+}, j_{-}\right]_{+}+\left[\mathcal{E} j^{\mathcal{E F}}, j_{+}\right]-\left[j^{\mathcal{F}}, j_{+}\right]-\left[j^{\mathcal{E F}}, j_{+}-j_{-}\right]_{+}, \\
\frac{\partial j_{-}}{\partial \tau} & =-j_{-}^{\prime}+2\left[j_{+}, j_{-}\right]_{-}+\left[\mathcal{E} j^{\mathcal{E F}}, j_{-}\right]+\left[j^{\mathcal{F}}, j_{-}\right]-\left[j^{\mathcal{E F}}, j_{+}-j_{-}\right]_{-}, \\
\frac{\partial j^{\mathcal{F}}}{\partial \tau} & =\mathcal{E}\left(j^{\mathcal{E F}}\right)^{\prime}+2\left[j_{+}, j_{-}\right]^{\mathcal{F}}+\left[\mathcal{E} j^{\mathcal{E F}}, j^{\mathcal{F}}\right]-\left[j^{\mathcal{E F}}, j_{+}-j_{-}\right]^{\mathcal{F}}, \\
\frac{\partial j^{\mathcal{E F}}}{\partial \tau} & =\left[\mathcal{E} j^{\mathcal{E F}}, j^{\mathcal{E F}}\right]-\left[j^{\mathcal{E F}}, j_{+}-j_{-}\right]^{\mathcal{E F}} .
\end{aligned}
$$


Imposing the reduction constraint $j^{\mathcal{E F}}=0$, we obtain the equations of motions of the degenerate $\hat{\mathcal{E}}$-model

$$
\begin{aligned}
& \frac{\partial j_{+}}{\partial \tau}=j_{+}^{\prime}+2\left[j_{+}, j_{-}\right]_{+}-\left[j^{\mathcal{F}}, j_{+}\right], \\
& \frac{\partial j_{-}}{\partial \tau}=-j_{-}^{\prime}+2\left[j_{+}, j_{-}\right]_{-}+\left[j^{\mathcal{F}}, j_{-}\right], \\
& \frac{\partial j^{\mathcal{F}}}{\partial \tau}=2\left[j_{+}, j_{-}\right]^{\mathcal{F}} .
\end{aligned}
$$

It is perhaps worth pointing out that the quantities $j_{ \pm}$and $j^{\mathcal{F}}$ appearing in the equation (3.18) are not well defined on the reduced phase space $L F \backslash L D^{c}$ because we find from the identity $j=l^{\prime} l^{-1}$ that they transform under the action of the loop group $L F$ as

$$
j_{ \pm} \rightarrow \operatorname{Ad}_{f} j_{ \pm}, \quad j^{\mathcal{F}} \rightarrow \operatorname{Ad}_{f} j^{\mathcal{F}}+\partial_{\sigma} f f^{-1}, \quad f \in L F
$$

However, the equations (3.18) themselves are invariant with respect to the transformation (3.19), they can be therefore indeed interpreted as the the evolution equations on the reduced phase space.

Remark 3.2. We note that the same set of the equations (3.18) can be obtained by identifying $A_{\sigma}=j^{\mathcal{F}}$ and by fixing the gauge $A_{\tau}=0$ in the following set of the $F$-gauge invariant equations (that is the gauge transformation depends also on $\tau$ )

$$
\begin{aligned}
\partial_{-} j_{+}-\left[A_{-}, j_{+}\right] & =2\left[j_{+}, j_{-}\right]_{+}, \\
\partial_{+} j_{-}-\left[A_{+}, j_{-}\right] & =2\left[j_{+}, j_{-}\right]_{-}, \\
\frac{1}{2} \partial_{-} A_{+}-\frac{1}{2} \partial_{+} A_{-}+\frac{1}{2}\left[A_{+}, A_{-}\right] & =2\left[j_{+}, j_{-}\right]^{\mathcal{F}},
\end{aligned}
$$

where

$$
\partial_{ \pm}:=\partial_{\tau} \pm \partial_{\sigma}, \quad A_{ \pm}:=A_{\tau} \pm A_{\sigma}
$$

The transformation (3.19) can be then interpreted as the residual time-independent gauge transformation preserving the gauge $A_{\tau}=0$.

Consider the degenerate $\hat{\mathcal{E}}$-model on the Drinfeld double $D$ and let $K \subset D$ be a half-dimensional subgroup such that the Lie subalgebra $\mathcal{K} \subset \mathcal{D}$ is isotropic with respect to the bilinear form $(., .)_{\mathcal{D}}$. Then there is a two-dimensional nonlinear $\sigma$-model such that its first order dynamics can be expressed in terms of this degenerate $\hat{\mathcal{E}}$-model; in particular its first order Hamiltonian equations of motion are given by (3.18). The action of this $\sigma$-model reads [32]

$$
\begin{gathered}
S_{\mathrm{WZW}}(l)=\frac{1}{4} \int \delta^{-1} \oint\left(\delta l l^{-1},\left[\partial_{\sigma} l l^{-1}, \delta l l^{-1}\right]_{\mathcal{D}}\right)_{\mathcal{D}}+ \\
+\frac{1}{4} \int d \tau \oint\left(W_{l}^{+} \partial_{+} l l^{-1}, \partial_{-} l l^{-1}\right)_{\mathcal{D}}-\frac{1}{4} \int d \tau \oint\left(\partial_{+} l l^{-1}, W_{l}^{-} \partial_{-} l l^{-1}\right)_{\mathcal{D}} .
\end{gathered}
$$


Here $W_{l}^{ \pm}: \mathcal{D} \rightarrow \mathcal{D}$ are the projectors fully characterized by their respective kernels and images

$$
\operatorname{Ker}\left(W_{l}^{ \pm}\right)=A d_{l}(\mathcal{K}), \quad \operatorname{Im}\left(W_{l}^{ \pm}\right)=V_{ \pm} \oplus \mathcal{F} .
$$

It may seem, that the $\sigma$-model (3.22) lives on the target $D$ but, actually, it lives on the space of double cosets $F \backslash D / K$ because it enjoys the gauge symmetries

$$
l(\tau, \sigma) \rightarrow f(\tau, \sigma) l(\tau, \sigma) k(\tau, \sigma), \quad f(\tau, \sigma) \in F, \quad k(\tau, \sigma) \in K .
$$

\subsection{Integrable dressing cosets}

Suppose that there is the degenerate $\hat{\mathcal{E}}$-model based on the Drinfeld double $D$, on the involution $\mathcal{E}$ and on the isotropic subalgebra $\mathcal{F} \subset \mathcal{D}$. Moreover, we suppose that there is a quadratic Lie algebra $\mathcal{G}$ which also possesses $\mathcal{F}$ as its subalgebra and that there is an one-parametric family of linear operators $\hat{O}(z): V_{+} \oplus V_{-} \rightarrow \mathcal{G}$ which intertwin the adjoint action of $\mathcal{F}$ on $V_{+} \oplus V_{-}$and on $\mathcal{G}$ and verify the following condition

$$
\left[\hat{O}(z) x_{+}, \hat{O}(z) x_{-}\right]_{\mathcal{G}}=\left[x_{+}, x_{-}\right]_{\mathcal{D}}^{\mathcal{F}}+\hat{O}(z)\left[x_{+}, x_{-}\right]_{\mathcal{D}}^{\perp}, \quad x_{ \pm} \in V_{ \pm} .
$$

Here the element $\left[x_{+}, x_{-}\right]_{\mathcal{D}}^{\mathcal{F}}$ of $\mathcal{F}$ is viewed as the element of $\mathcal{G}$.

It turns out that the condition (3.25) is sufficient for the weak Lax integrability

of the degenerate $\hat{\mathcal{E}}$-model. Indeed, the Lax pair $L(z), M(z)$ of the operators acting on the loop Lie algebra $L \mathcal{G}$ is given by the formulas

$$
\begin{gathered}
L(z)=\partial_{\sigma}-\operatorname{ad}_{j^{\mathcal{F}}+\hat{O}(z) j^{\perp}}^{\mathcal{G}} \\
M(z)=-\operatorname{ad}_{\hat{O}(z) \mathcal{E} j^{\perp}}^{\mathcal{G}}
\end{gathered}
$$

as it is straightforward to find out from (3.25) that the dressing coset field equations (3.18) of the degenerate $\hat{\mathcal{E}}$-model can be indeed represented in the Lax form with the spectral parameter

$$
\frac{d L(z)}{d t}=[L(z), M(z)]
$$

To guarantee the strong Lax integrability of the degenerate $\hat{\mathcal{E}}$-model, we first define

$$
O(z)\left(j^{\mathcal{F}}+j^{\perp}+j^{\mathcal{E F}}\right):=j^{\mathcal{F}}+\hat{O}(z) j^{\perp}
$$

and we supplement the condition (3.25) with the condition that it exists a family of operators $\hat{r}(z, w): \mathcal{G} \rightarrow \mathcal{G}$ such that it holds

$$
\begin{gathered}
{\left[O^{\dagger}(z) x, O^{\dagger}(w) y\right]_{\mathcal{D}}+O^{\dagger}(z)[x, \hat{r}(z, w) y]_{\mathcal{G}}+O^{\dagger}(w)[\hat{r}(w, z) x, y]_{\mathcal{G}} \in \mathcal{F}, \quad \forall x, y \in \mathcal{G},} \\
\left(O^{\dagger}(z) x, O^{\dagger}(w) y\right)_{\mathcal{D}}+(x, \hat{r}(z, w) y)_{\mathcal{G}}+(\hat{r}(w, z) x, y)_{\mathcal{G}}=0, \quad \forall x, y \in \mathcal{G} .
\end{gathered}
$$

Here $O^{\dagger}(z): \mathcal{G} \rightarrow \mathcal{D}$ is the adjoint of the operator $O(z)$ defined by the relation

$$
(O(z) x, y)_{\mathcal{G}}=\left(x, O^{\dagger}(z) y\right)_{\mathcal{D}}, \quad \forall x \in \mathcal{D}, y \in \mathcal{G} .
$$


Let us now see why the conditions (3.30) and (3.31) guarantee the strong integrability. First we remark that the operators $\hat{O}(z)$ were supposed to intertwin the adjoint action of $\mathcal{F}$ on $V_{+} \oplus V_{-}$and on $\mathcal{G}$ which implies that the Lax operator $L(z)$ transforms in the adjoint way upon the action of the loop group $L F$. Indeed, using Eq. (3.19), we find

$$
\begin{gathered}
L^{f}(z):=\partial_{\sigma}-\operatorname{ad}_{\operatorname{Ad}_{f}^{D} j^{\mathcal{F}}+\partial_{\sigma} f f^{-1}+\hat{O}(z) \operatorname{Ad}_{f}^{D} j^{\perp}}^{\mathcal{G}}= \\
=\operatorname{Ad}_{f}^{G}\left(\partial_{\sigma}-\operatorname{ad}_{j^{\mathcal{F}}+\hat{O}(z) j^{\perp}}^{\mathcal{G}}\right) \operatorname{Ad}_{f^{-1}}^{G}=\operatorname{Ad}_{f}^{G} L(z) \operatorname{Ad}_{f^{-1}}^{G} .
\end{gathered}
$$

We thus observe that the spectral invariants of the Lax matrix $L(z)$ are invariant with respect to the action of the loop group $L F$ and therefore they restrict to the well defined functions on the reduced phase space. Moreover, by the general theory of symplectic reduction, the reduced Poisson brackets of the restricted spectral invariants can be evaluated by restricting the unreduced Poisson brackets of the unrestricted invariants. In particular, if the unreduced brackets vanish on the constraint surface, the reduced ones vanish. Said in other words, the reduced spectral invariants (which are automatically the integrals of the reduced motion) Poissoncommute if the unreduced Poisson brackets of the unreduced spectral invariants vanish on the constraint surface $j^{\mathcal{E F}}=0$. This occurs precisely if the following condition holds

$$
\{L(z) \otimes \mathrm{Id}, \mathrm{Id} \otimes L(w)\}_{\text {unred }} \approx[r(z, w), L(z) \otimes \mathrm{Id}]-\left[r^{p}(w, z), \operatorname{Id} \otimes L(w)\right] .
$$

Here $r(z, w)$ is some $r$-matrix acting on the space $L \mathcal{G} \otimes L \mathcal{G}$ and the symbol $\approx$ indicates that the left-hand-side is equal to the right-hand-side on the constraint surface $j^{\mathcal{E F}}=0$. Said in other words, we first evaluate the unreduced brackets of the Lax operator with itself, then we restrict the result to the constraint surface $j^{\mathcal{E F}}=0$ and we require that the succession of these two operations gives the right-hand-side of (3.34) also restricted to the constraint surface.

If we choose the ansatz (2.19)

$$
r(z, w)=C_{A B} \operatorname{ad}_{\hat{r}(z, w) T^{A}} \otimes \operatorname{ad}_{T^{B}} \delta\left(\sigma_{1}-\sigma_{2}\right),
$$

then, similarly as in Section 2.3, we deduce from the conditions (3.30) and (3.31) the validity of the strong integrability condition (3.34). Indeed, we use the current Poisson bracket (2.3) to calculate the unreduced Poisson brackets of the matrix elements of the Lax operator

$$
\begin{gathered}
\left\{\left(O(z) j\left(\sigma_{1}\right), x\right)_{\mathcal{G}},\left(O(w) j\left(\sigma_{2}\right), y\right)_{\mathcal{G}}\right\}_{\text {unred }}= \\
=\left(j\left(\sigma_{1}\right),\left[O^{\dagger}(z) x, O^{\dagger}(w) y\right]_{\mathcal{D}}\right)_{\mathcal{D}} \delta\left(\sigma_{1}-\sigma_{2}\right)+\left(O^{\dagger}(z) x, O^{\dagger}(w) y\right)_{\mathcal{D}} \partial_{\sigma_{1}} \delta\left(\sigma_{1}-\sigma_{2}\right)= \\
=-\left(j\left(\sigma_{1}\right), O^{\dagger}(z)[x, \hat{r}(z, w) y]_{\mathcal{G}}+O^{\dagger}(w)[\hat{r}(w, z) x, y]_{\mathcal{G}}+\phi\right)_{\mathcal{D}} \delta\left(\sigma_{1}-\sigma_{2}\right) \\
-\left((x, \hat{r}(z, w) y)_{\mathcal{G}}+(\hat{r}(w, z) x, y)_{\mathcal{G}}\right) \partial_{\sigma_{1}} \delta\left(\sigma_{1}-\sigma_{2}\right) .
\end{gathered}
$$


Here $\phi$ as an unspecified element of $\mathcal{F} \subset \mathcal{D}$ which is eventually irrevelant because

$$
\left(j\left(\sigma_{1}\right), \phi\right)_{\mathcal{D}}=\left(j^{\mathcal{E F}}\left(\sigma_{1}\right), \phi\right)_{\mathcal{D}} \approx 0
$$

We conclude the argument by remarking that the desired relation (3.34) is nothing but the operator form of the equation (3.36).

\section{$4 \quad \sigma$-models on symmetric spaces}

In the present section, we test succesfully our method by applying it to three $\sigma$ models on symmetric spaces for which the strong integrability has been already proven in the literature. We first represent all those three models as the dressing cosets and then we readily formulate and solve for them the integrability conditions (3.25), (3.30) and (3.31). Our unified treatment does not give exactly the same Lax matrices (nor, for that matter, the same $r$-matrices) that the ones obtained in literature previously, however, our Lax matrices differ from those previously known just outside of the primary constraints surface. This means that they give the same set of the integral of motion in involution as the old Lax matrices do.

\subsection{The non-deformed symmetric space $\sigma$-model}

Let $\rho: G \rightarrow G$ be an involutive automorphism of a quadratic Lie group $G$ and denote $H$ the subgroup of $G$ consisting of fixed points of this automorphism. The tangent involutive automorphism $\rho_{*}: \mathcal{G} \rightarrow \mathcal{G}$ has two eigenspaces: to the eigenvalue +1 it corresponds the Lie algebra $\mathcal{H}$ and to the eigenvalue -1 it corresponds an $\operatorname{ad}_{\mathcal{H}}$-module $\mathcal{H}^{\perp}$. As the notation suggests, the eigenspaces $\mathcal{H}$ and $\mathcal{H}^{\perp}$ are orthogonal to each other with respect to the ad-invariant non-degenerate symmetric bilinear form $(., .)_{\mathcal{G}}$ on $\mathcal{G}$. We note also that the Lie bracket of two elements from $\mathcal{H}^{\perp}$ belongs to $\mathcal{H}$.

The space of left cosets $H \backslash G$ is referred to as the symmetric space. We now construct the dressing coset which gives rise to an integrable $\sigma$-model living on the symmetric space target $H \backslash G$. For the Drinfeld double $D$ we take the cotangent bundle $T^{*} G$ and we view the elements of $D$ as pairs $(g, \gamma), g \in G, \gamma \in \mathcal{G}$. The group multiplication law then reads

$$
(g, x)(m, y)=\left(g m, x+\operatorname{Ad}_{g} y\right), \quad g, m \in G, \quad x, y \in \mathcal{G} .
$$

Furthermore, the unit element $e_{D}$ of $D=T^{*} G$ is

$$
e_{D}=\left(e_{G}, 0\right)
$$

and the inverse element is

$$
(g, x)^{-1}=\left(g^{-1},-A d_{g^{-1}} x\right)
$$


The involutive automorphism $\rho: G \rightarrow G$ can be naturally lifted to the involutive automorphism $\rho_{D}: D \rightarrow D$ by the formula

$$
\rho_{D}(g, x):=\left(\rho(g), \rho_{*} x\right)
$$

We denote by $D_{H}$ the subgroup of the fixed points of the automorphism $\rho_{D}$. The elements of $D_{H}$ are obviously the pairs $(h, u), h \in H, u \in \mathcal{H}$.

The elements of the Lie algebra $\mathcal{D}$ are pairs $\left(\xi_{0}, \xi_{1}\right), \xi_{i} \in \mathcal{G}, i=0,1$, with the Lie bracket given by

$$
\left[\left(\xi_{0}, \xi_{1}\right),\left(\chi_{0}, \chi_{1}\right)\right]_{\mathcal{D}}=\left(\left[\xi_{0}, \chi_{0}\right]_{\mathcal{G}},\left[\xi_{0}, \chi_{1}\right]_{\mathcal{G}}+\left[\xi_{1}, \chi_{0}\right]_{\mathcal{G}}\right)
$$

The symmetric non-degenerate ad-invariant bilinear form $(., .)_{\mathcal{D}}$ on the Lie algebra $\mathcal{D}$ is given by

$$
\left(\left(\xi_{0}, \xi_{1}\right),\left(\chi_{0}, \chi_{1}\right)\right)_{\mathcal{D}}=\left(\xi_{0}, \chi_{1}\right)_{\mathcal{G}}+\left(\xi_{1}, \chi_{0}\right)_{\mathcal{G}}
$$

where $(., .)_{\mathcal{G}}$ is the symmetric non-degenerate ad-invariant bilinear form on $\mathcal{G}$. In this paper, $\mathcal{G}$ will always be semi-simple and $(., .)_{\mathcal{G}}$ its standard (negatively definite) Killing-Cartan form. Note also that the form $(., .)_{\mathcal{D}}$ has the split signature $(+, \ldots,+,-, \ldots,-)$.

We shall also need formulas for the left and right Maurer-Cartan forms on $D$

$$
\begin{gathered}
l^{-1} d l \equiv(g, x)^{-1} d(g, x)=\left(g^{-1} d g, \operatorname{Ad}_{g^{-1}}(d x)\right), \\
d l l^{-1} \equiv d(g, x)(g, x)^{-1}=\left(d g g^{-1}, d x+\left[x, d g g^{-1}\right]\right),
\end{gathered}
$$

and the formula expressing the adjoint action of $D$ on $\mathcal{D}$

$$
\operatorname{Ad}_{(g, x)}\left(\xi_{0}, \xi_{1}\right)=\left(\operatorname{Ad}_{g} \xi_{0}, \operatorname{Ad}_{g} \xi_{1}+\operatorname{ad}_{x} \operatorname{Ad}_{g} \xi_{0}\right)
$$

Now we define the non-degenerate $\mathcal{E}$-model on $D$ by considering an involution $\mathcal{E}: \mathcal{D} \rightarrow \mathcal{D}$ defined as

$$
\mathcal{E}\left(\xi_{0}, \xi_{1}\right)=-\left(\xi_{1}, \xi_{0}\right)
$$

This involution verifies all required properties, it is symmetric with respect to the bilinear form $(.,)_{\mathcal{D}}$ and the bilinear form $(., \mathcal{E} \text {. })_{\mathcal{D}}$ is strictly positive definite. Indeed, we have

$$
\left(\left(\xi_{0}, \xi_{1}\right), \mathcal{E}\left(\xi_{0}, \xi_{1}\right)\right)_{\mathcal{D}}=-\left(\xi_{0}, \xi_{0}\right)_{\mathcal{G}}-\left(\xi_{1}, \xi_{1}\right)_{\mathcal{G}}
$$

In order, to define the degenerate $\hat{\mathcal{E}}$-model we need the isotropic subgroup $F \subset D$ such that the adjoint action of $F$ on $\mathcal{D}$ commutes with the involution $\mathcal{E}$. We choose for $F$ the group $H$, viewed as the subgroup of $D$. We can now identify the subspaces featuring in the decomposition (3.3) of the Drinfeld double $\mathcal{D}$ :

$$
(\phi, 0) \in \mathcal{F}, \quad(0, \phi) \in \mathcal{E} \mathcal{F}, \quad(\xi, \mp \xi) \in V_{ \pm}, \quad \phi \in \mathcal{H}, \quad \xi \in \mathcal{H}^{\perp}
$$


In order to obtain the $\sigma$-model action (3.22) corresponding to our degenerate $\hat{\mathcal{E}}$-model, we have to choose the half-dimensional isotropic subgroup $K \subset D$. We choose for $K$ the vector space $\mathcal{G}$ viewed as the Abelian Lie group the elements of which have the form $\left(e_{G}, \gamma\right) \in D$. We fix the $K$-part of the gauge symmetry (3.24) by setting $l=g \in G$, we then find

$$
W_{g}^{ \pm}\left(\partial_{ \pm} g g^{-1}, 0\right)=\left(\partial_{ \pm} g g^{-1}, \mp P^{\perp} \partial_{ \pm} g g^{-1}\right)
$$

and, finally, we conclude from (3.22)

$$
S_{\hat{\mathcal{E}}}(g)=-\frac{1}{2} \int d \tau \oint\left(P^{\perp} \partial_{+} g g^{-1}, P^{\perp} \partial_{-} g g^{-1}\right)_{\mathcal{G}} .
$$

Here $P^{\perp}: \mathcal{G} \rightarrow \mathcal{H}^{\perp}$ is the projector with the kernel $\mathcal{H}$. We notice that the action (4.14) has the residual gauge symmetry $g \rightarrow h g$, it therefore lives indeed on the symmetric space target $H \backslash G$.

Now we study the integrability of the dressing coset (4.14). Following the analysis of Section 3.2, we look for the families of operators $\hat{O}(z)$ and $\hat{r}(z, w)$ verifying the conditions (3.25), (3.30) and (3.31) of Section 3.2. We choose the ansatz given by the multiplication by the numerical functions

$$
\begin{gathered}
\hat{O}(z)(\xi, \mp \xi)=a_{ \pm}(z) \xi, \quad \xi \in \mathcal{H}^{\perp} ; \\
\hat{r}(z, w) \phi=b(z, w) \phi, \quad \hat{r}(z, w) \xi=c(z, w) \xi, \quad \phi \in \mathcal{H}, \quad \xi \in \mathcal{H}^{\perp} .
\end{gathered}
$$

We immediately observe that the choice (4.15) respects the condition (3.25) provided it holds

$$
a_{+}(z) a_{-}(z)=1
$$

The dressing coset (4.14) is therefore at least weakly integrable; we may choose without loss of generality

$$
a_{ \pm}(z)=z^{ \pm 1}
$$

With the choice (4.18), we find the operators $O^{\dagger}(z)$

$$
O^{\dagger}(z) \phi=(0, \phi), \quad \phi \in \mathcal{H}, \quad O^{\dagger}(z) \xi=\frac{1}{2 z}(\xi, \xi)-\frac{z}{2}(\xi,-\xi), \quad \xi \in \mathcal{H}^{\perp}
$$

The conditions (3.30) and (3.31) then give

$$
\begin{aligned}
b(z, w)+b(w, z) & =0 \\
c(z, w)+c(w, z) & =\frac{1}{2} u(z w) \\
b(z, w) z^{ \pm 1}+c(w, z) w^{ \pm 1} & =\frac{1}{2} u(z),
\end{aligned}
$$

where

$$
u(x):=x-x^{-1} .
$$


The system (4.20) does have a(n unique) solution given by

$$
b(z, w)=\frac{1}{2} \frac{u(z) u(w)}{u(w / z)}, \quad c(z, w)=\frac{1}{2} \frac{u(w)^{2}}{u(w / z)},
$$

we thus conclude that the symmetric space dressing coset (4.14) is strongly integrable.

Remark 4.1. The strong integrability of the symmetric space $\sigma$-model (4.14) was already established in Refs. $[9,10]$. The Lax matrix of those references is not the same as ours, however, it differs from ours only outside the constraint surface $j^{\mathcal{E F}}=0$ (or $\Pi^{(0)}=0$ in the notation of $\left.[9,10]\right)$. This means that the reduced spectral invariants of our Lax matrix coincide with those of the Lax matrix of Refs. [9, 10]. It may be said also that our Lax matrix (2.10) is simpler than that of Refs. $[9,10]$ because it does not contain the variable $j^{\mathcal{E F}}$ but the price to pay for it is that our $r$-matrix $\hat{r}(\lambda, \eta)$ is more complicated than that of Ref. $[9,10]$ because it is not given by a multiple of identity.

\subsection{Symmetric space $\lambda$-deformation}

As in the previous section, let $\rho: G \rightarrow G$ be the involutive automorphism of a semi-simple Lie group $G$ and denote $H$ the subgroup of $G$ consisting of fixed points of this automorphism. However, for the double $D$ we now take the direct product $D=G \times G$ and for the automorphism $\rho_{D}$ we take

$$
\rho_{D}\left(a_{L}, a_{R}\right):=\left(\rho\left(a_{L}\right), \rho\left(a_{R}\right)\right), \quad\left(a_{L}, a_{R}\right) \in G \times G .
$$

The symmetric non-degenerate ad-invariant bilinear form $(., .)_{\mathcal{D}}$ on the Lie algebra $\mathcal{D}$ is now given by

$$
\left(\left(\mu_{L}, \mu_{R}\right),\left(\nu_{L}, \nu_{R}\right)\right)_{\mathcal{D}}=\left(\mu_{L}, \nu_{L}\right)_{\mathcal{G}}-\left(\mu_{R}, \nu_{R}\right)_{\mathcal{G}}, \quad \mu_{L, R}, \nu_{L, R} \in \mathcal{G}
$$

where $(., .)_{\mathcal{G}}$ is the (negatively definite) Killing-Cartan form as in Section 4.1. Again, the form $(., .)_{\mathcal{D}}$ has the split signature $(+, \ldots,+,-, \ldots,-)$.

Now we define the non-degenerate $\mathcal{E}$-model on $D$ by considering an involution $\mathcal{E}_{\alpha}: \mathcal{D} \rightarrow \mathcal{D}$ defined as

$$
\mathcal{E}_{\alpha}\left(\mu_{L}, \mu_{R}\right)=\cosh \alpha\left(-\mu_{L}, \mu_{R}\right)+\sinh \alpha\left(-\mu_{R}, \mu_{L}\right) .
$$

This involution verifies all required properties, it is symmetric with respect to the bilinear form $(., .)_{\mathcal{D}}$ and the bilinear form $\left(., \mathcal{E}_{\alpha} \cdot\right)_{\mathcal{D}}$ is strictly positive definite. Indeed, we have

$$
\left((\mu, \nu), \mathcal{E}_{\alpha}(\mu, \nu)\right)_{\mathcal{D}}=-\frac{e^{\alpha}}{2}(\mu+\nu, \mu+\nu)_{\mathcal{G}}-\frac{e^{-\alpha}}{2}(\mu-\nu, \mu-\nu)_{\mathcal{G}}, \quad \mu, \nu \in \mathcal{G}
$$

In order to define the degenerate $\hat{\mathcal{E}}$-model we need the isotropic subgroup $F \subset$ $D$ such that the adjoint action of $F$ on $\mathcal{D}$ commutes with the involution $\mathcal{E}_{\alpha}$. We choose for $F$ the subgroup

$$
F=\{(f, f) \in D, \quad f \in H\}
$$


where, as in Section 4.1, $H$ is the subgroup of $G$ consisting of the fixed points of the involutive automorphism $\rho: G \rightarrow G$. We can now identify the subspaces featuring in the decomposition (3.3) of the Drinfeld double $\mathcal{D}$ :

$$
(\phi, \phi) \in \mathcal{F}, \quad(-\phi, \phi) \in \mathcal{E} \mathcal{F}, \quad\left(\lambda^{ \pm 1} \xi, \xi\right) \in V_{ \pm}, \quad \phi \in \mathcal{H}, \quad \xi \in \mathcal{H}^{\perp}
$$

where

$$
\lambda:=\frac{1-e^{\alpha}}{1+e^{\alpha}}
$$

In order to obtain the $\sigma$-model action (3.22) corresponding to our $\lambda$-deformed degenerate $\hat{\mathcal{E}}$-model, we have to choose the half-dimensional isotropic subgroup $K \subset D$. We choose for $K$ the diagonal subgroup of $D$, which means

$$
K=\{(x, x) \in D, \quad x \in G\}
$$

We fix the $K$-part of the gauge symmetry (3.24) by setting $l_{0}=\left(g, e_{G}\right) \in D$, we then find

$$
\begin{gathered}
W_{g}^{ \pm} \partial_{ \pm} l_{0} l_{0}^{-1}=W_{g}^{ \pm}\left(\partial_{ \pm} g g^{-1}, 0\right)= \\
=-\left(\left(\lambda^{ \pm 1} P^{\perp}+P\right)\left(\operatorname{Ad}_{g}-\lambda^{ \pm 1} P^{\perp}-P\right)^{-1} \partial_{ \pm} g g^{-1},\left(\operatorname{Ad}_{g}-\lambda^{ \pm 1} P^{\perp}-P\right)^{-1} \partial_{ \pm} g g^{-1}\right)
\end{gathered}
$$

Here $P: \mathcal{G} \rightarrow \mathcal{H}$ is the projector with the kernel $\mathcal{H}^{\perp}$. Finally, we conclude from $(3.22)$

$$
\begin{array}{rl}
S_{\lambda}(g)=\frac{1}{4} \int d & d\left(\partial_{+} g g^{-1}, \partial_{-} g g^{-1}\right)_{\mathcal{G}}+\frac{1}{4} \int \delta^{-1} \oint\left(\delta g g^{-1},\left[\partial_{\sigma} g g^{-1}, \delta g g^{-1}\right]\right)_{\mathcal{G}} \\
+ & \frac{1}{2} \int d \tau \oint\left(\partial_{+} g g^{-1}, \frac{1}{\mathrm{Ad}_{g}\left(\lambda P^{\perp}+P\right)-1} \partial_{-} g g^{-1}\right)_{\mathcal{G}}
\end{array}
$$

We notice that the action (4.32) has the residual gauge symmetry $g \rightarrow h g h^{-1}$, $h \in H$.

Now we study the integrability of the dressing coset (4.32). Following the analysis of Section 3.2, we look for the families of operators $\hat{O}(z)$ and $\hat{r}(z, w)$ verifying the conditions (3.25), (3.30) and (3.31) of Section 3.2. We choose the ansatz given by the multiplication by the numerical functions

$$
\begin{gathered}
\hat{O}(z)\left(\lambda^{ \pm 1} \xi, \xi\right)=a_{ \pm}(z) \xi, \quad \xi \in \mathcal{H}^{\perp} ; \\
\hat{r}(z, w) \phi=b(z, w) \phi, \quad \hat{r}(z, w) \xi=c(z, w) \xi, \quad \phi \in \mathcal{H}, \quad \xi \in \mathcal{H}^{\perp} .
\end{gathered}
$$

We immediately observe that the choice (4.33) respects the condition (3.25) provided it holds

$$
a_{+}(z) a_{-}(z)=1
$$

The dressing coset (4.32) is therefore at least weakly integrable; we may choose without loss of generality

$$
a_{ \pm}(z)=\lambda^{ \pm \frac{1}{2}} z^{ \pm 1} .
$$


With the choice (4.36), we find the operators $O^{\dagger}(z)$

$$
O^{\dagger}(z)(\phi+\xi)=\left(\frac{1}{2} \phi+\frac{u\left(z \lambda^{\frac{1}{2}}\right)}{u(\lambda)} \xi,-\frac{1}{2} \phi+\frac{u\left(z \lambda^{-\frac{1}{2}}\right)}{u(\lambda)} \xi\right), \quad \phi \in \mathcal{H}, \xi \in \mathcal{H}^{\perp},
$$

where

$$
u(x):=x-x^{-1} .
$$

The conditions (3.30) and (3.31) then give

$$
\begin{aligned}
b(z, w)+b(w, z) & =0 \\
c(z, w)+c(w, z) & =-\frac{u(z w)}{u(\lambda)} \\
b(z, w) u\left(z \lambda^{ \pm \frac{1}{2}}\right)+c(w, z) u\left(w \lambda^{ \pm \frac{1}{2}}\right) & =\mp \frac{1}{2} u\left(z \lambda^{ \pm \frac{1}{2}}\right) .
\end{aligned}
$$

The system (4.39) does have a(n unique) solution given by

$$
b(z, w)=\frac{u\left(z \lambda^{-\frac{1}{2}}\right) u\left(w \lambda^{\frac{1}{2}}\right)}{u(\lambda) u(z / w)}-\frac{1}{2}, \quad c(z, w)=\frac{u\left(w \lambda^{-\frac{1}{2}}\right) u\left(w \lambda^{\frac{1}{2}}\right)}{u(\lambda) u(z / w)},
$$

we thus conclude that the $\lambda$-deformed symmetric space dressing coset (4.32) is strongly integrable.

Remark 4.2. The strong integrability of the symmetric space $\lambda$-deformation (4.32) was already established in Refs. [17,22]. The Lax matrix of those references is not the same as ours, but, again, it differs from ours only outside the constraint surface $j^{\mathcal{E F}}=0$, which means that the reduced spectral invariants of our Lax matrix coincide with those coming from the Lax matrix of Refs. [17,22].

\subsection{Symmetric space $\eta$-deformation}

Let $\rho: G \rightarrow G$ be the involutive automorphism of the semi-simple Lie group $G$ and denote $H$ the subgroup of $G$ consisting of fixed points of this automorphism. Suppose moroever, that $\rho$ can be lifted to an involutive automorphism of the complexified group $\rho_{\mathbb{C}}: G^{\mathbb{C}} \rightarrow G^{\mathbb{C}}$. For the Drinfeld double we then take the Lu-Weinstein one [39] which is the complexified group $D=G^{\mathbb{C}}$ viewed as the real group of doubled dimension. The symmetric non-degenerate ad-invariant bilinear form $(., .)_{\mathcal{D}}$ on the Lie algebra $\mathcal{D}$ is now given by an expression which depends on a real positive parameter $\eta$

$$
\left(\mu_{1}+\mathrm{i} \nu_{1}, \mu_{2}+\mathrm{i} \nu_{2}\right)_{\mathcal{D}}=\frac{1}{\eta}\left(\mu_{1}, \nu_{2}\right)_{\mathcal{G}}+\frac{1}{\eta}\left(\mu_{2}, \nu_{1}\right)_{\mathcal{G}}, \quad \mu_{1,2}, \nu_{1,2} \in \mathcal{G} .
$$

Again, the form $(., .)_{\mathcal{D}}$ turns out to have the split signature $(+, \ldots,+,-, \ldots,-)$.

Now we define the non-degenerate $\mathcal{E}$-model on $D$ by considering an involution $\mathcal{E}_{\eta}: \mathcal{D} \rightarrow \mathcal{D}$ defined as

$$
\mathcal{E}_{\eta}(\mu+\mathrm{i} \nu)=-\eta^{-1} \nu-\mathrm{i} \eta \mu, \quad \eta>0, \quad \mu, \nu \in \mathcal{G} .
$$


This involution verifies all required properties, it is symmetric with respect to the bilinear form $(., .)_{\mathcal{D}}$ and the bilinear form $\left(., \mathcal{E}_{\eta} .\right)_{\mathcal{D}}$ is strictly positive definite. Indeed, we have

$$
\left(\mu+\mathrm{i} \nu, \mathcal{E}_{\eta}(\mu+\mathrm{i} \nu)_{\mathcal{D}}=-(\mu, \mu)_{\mathcal{G}}-\eta^{-2}(\nu, \nu)_{\mathcal{G}}, \quad \mu, \nu \in \mathcal{G}\right.
$$

In order to define the degenerate $\hat{\mathcal{E}}$-model, we choose for the isotropic subgroup $F$ the subgroup $H \subset G \subset G^{\mathbb{C}}$. The adjoint action of $F$ on $\mathcal{D}=\mathcal{G}^{\mathbb{C}}$ then indeed commutes with the involution $\mathcal{E}_{\eta}$. We can now identify the subspaces featuring in the decomposition (3.3) of the Drinfeld double $\mathcal{D}$ :

$$
\phi \in \mathcal{F}, \quad \mathrm{i} \phi \in \mathcal{E} \mathcal{F}, \quad \xi \mp \mathrm{i} \eta \xi \in V_{ \pm}, \quad \phi \in \mathcal{H}, \quad \xi \in \mathcal{H}^{\perp} .
$$

In order to obtain the $\sigma$-model action (3.22) corresponding to our $\eta$-deformed degenerate $\hat{\mathcal{E}}$-model, we have to choose the half-dimensional isotropic subgroup $K \subset D$. We choose for $K$ the subgroup $A N$ of $G^{\mathbb{C}}$ featuring in the Iwasava decomposition ${ }^{1} G^{\mathbb{C}}=G A N$.

In what follows; it will be convenient to parametrize the elements of the Lie algebra $\mathcal{K}$ in terms of those of the Lie algebra $\mathcal{G}$. This can be achieved via the $\mathbb{R}$-linear Yang-Baxter operator $R: \mathcal{G}^{\mathbb{C}} \rightarrow \mathcal{G}^{\mathbb{C}}$ defined as

$$
R E^{\alpha}=-\operatorname{sign}(\alpha) \mathrm{i} E^{\alpha}, \quad R H^{j}=0, \quad[R, \mathrm{i}]=0,
$$

where $E^{\alpha}, H^{j}$ is the standard Chevalley basis of $\mathcal{G}^{\mathbb{C}}$ and $\mathrm{i}$ is viewed as the operator of multiplication by the imaginary unit. Indeed, every element of $\mathcal{K}$ can be then written in an unique way as $(R-\mathrm{i}) \chi$, where $\chi \in \mathcal{G}$. We now fix the $K$-part of the gauge symmetry (3.24) by setting $l_{0}=g \in G$, we then find

$W_{g}^{ \pm} \partial_{ \pm} l_{0} l_{0}^{-1}=W_{g}^{ \pm} \partial_{ \pm} g g^{-1}=\partial_{ \pm} g g^{-1}-\eta\left(R_{g^{-1}}-\mathrm{i}\right)\left(\eta P^{\perp} R_{g^{-1}} P^{\perp} \mp 1_{\mathcal{H}^{\perp}}\right)^{-1} P^{\perp} \partial_{ \pm} g g^{-1}$,

where

$$
R_{g^{-1}}=\operatorname{Ad}_{g} R \operatorname{Ad}_{g^{-1}}
$$

Finally, we conclude from (3.22)

$$
S_{\eta}(g)=-\frac{1}{2} \int d \tau \oint\left(P^{\perp} \partial_{+} g g^{-1},\left(1_{\mathcal{H}^{\perp}}+\eta P^{\perp} R_{g^{-1}} P^{\perp}\right)^{-1} P^{\perp} \partial_{-} g g^{-1}\right)_{\mathcal{G}}
$$

We notice that the action (4.48) is the one-parameter deformation of the action (4.14) and it has also the residual gauge symmetry $g \rightarrow h g, h \in H$.

Now we study the integrability of the $\eta$-deformed dressing coset (4.48). Following the analysis of Section 3.2, we look for the families of operators $\hat{O}(z)$ and

\footnotetext{
${ }^{1}$ Recall, that $A$ is the non-compact part of the complexified Cartan torus of $G$ and $N$ is the nilpotent subgroup generated by the positive roots. In particular, if $G^{\mathbb{C}}$ is $S L(N, \mathbb{C})$, then $A N$ consists of uppertriangular matrices with real positive diagonal.
} 
$\hat{r}(z, w)$ verifying the conditions (3.25), (3.30) and (3.31) of Section 3.2. We choose the ansatz given by the multiplication by the numerical functions

$$
\begin{gathered}
\hat{O}(z) \frac{1 \mp \mathrm{i} \eta}{\sqrt{1+\eta^{2}}} \xi=a_{ \pm}(z) \xi, \quad \xi \in \mathcal{H}^{\perp} ; \\
\hat{r}(z, w) \phi=b(z, w) \phi, \quad \hat{r}(z, w) \xi=c(z, w) \xi, \quad \phi \in \mathcal{H}, \quad \xi \in \mathcal{H}^{\perp} .
\end{gathered}
$$

We immediately observe that the choice (4.49) respects the condition (3.25) provided it holds

$$
a_{+}(z) a_{-}(z)=1
$$

The dressing coset (4.48) is therefore at least weakly integrable; we may choose without loss of generality

$$
a_{ \pm}(z)=z^{ \pm 1}
$$

With the choice (4.52), we find the operators $O^{\dagger}(z)$

$$
O^{\dagger}(z)(\phi+\xi)=\mathrm{i} \eta \phi+\frac{1}{2} \sqrt{1+\eta^{2}}\left((1+\mathrm{i} \eta) z^{-1}-(1-\mathrm{i} \eta) z\right) \xi, \quad \phi \in \mathcal{H}, \xi \in \mathcal{H}^{\perp} .
$$

The conditions (3.30) and (3.31) then give

$$
\begin{aligned}
b(z, w)+b(w, z) & =0 \\
c(z, w)+c(w, z) & =\frac{1+\eta^{2}}{2} u(z w) \\
b(z, w) z^{ \pm 1}+c(w, z) w^{ \pm 1} & =\frac{1}{2} u(z) \mp \frac{\eta^{2}}{2} s(z)
\end{aligned}
$$

where

$$
u(x):=x-x^{-1}, \quad s(x):=x+x^{-1} .
$$

The system (4.54) does have a(n unique) solution given by

$$
b(z, w)=\frac{1}{2} \frac{u(z) u(w)+\eta^{2} s(z) s(w)}{u(w / z)}, \quad c(z, w)=\frac{1}{2} \frac{u(w)^{2}+\eta^{2} s(w)^{2}}{u(w / z)},
$$

we thus conclude that the $\eta$-deformed symmetric space dressing coset (4.48) is strongly integrable.

Remark 4.3. The strong integrability of the symmetric space $\eta$-deformation (4.48) was already established in Ref. [10]. The Lax matrix of those references is not the same

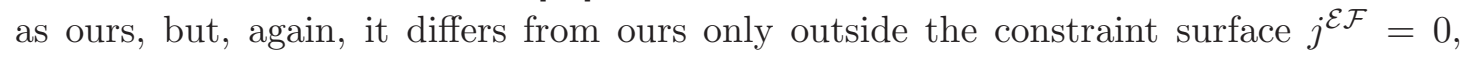
which means that the reduced spectral invariants of our Lax matrix coincide with those coming from the Lax matrix of Ref. [10]. 


\section{Generalized pseudo-chiral models}

In all three examples of the integrable dressing cosets treated in Section 4, the second term on the right-hand-side of the condition (3.25) vanished identically. Now we are going to consider examples where it is no longer the case. The simplest one of those new integrable examples is the pseudo-chiral $\sigma$-model of Zakharov and Mikhailov [53] characterized by the action

$$
S_{\mu}(x)=-\frac{1}{2 \mu} \int d \tau \oint\left(\left(\partial_{+} x, \partial_{-} x\right)_{\mathcal{G}}+\frac{\mu}{3}\left(x,\left[\partial_{+} x, \partial_{-} x\right]_{\mathcal{G}}\right)_{\mathcal{G}}\right) .
$$

Here the field $x(\tau, \sigma)$ takes values in the Lie algebra $\mathcal{G}$.

In the present section, we first interpret the pseudo-chiral model (5.57) as the dressing coset based on the Drinfeld double ${ }^{2} D=J^{3} G$, where $J^{3} G$ is the $3^{\text {rd }}$ order jet bundle of the semi-simple Lie group $G$. We then obtain more complicated integrable dressing cosets by considering higher order jet bundles $J^{2 n+1} G$. Those theories are apparently new and they describe an integrable interaction of $n \mathcal{G}$ valued fields. They represent the principal examples where our method does not just confirm the old results but provides genuinely new ones.

\subsection{Pseudo-chiral model}

Consider the Drinfeld double $D=J^{3} G$ introduced in [37], where $J^{3} G$ is the $3^{\text {rd }}$ order jet bundle of the quadratic Lie group $G$. The group $J^{3} G$ can be conveniently parametrized via the right trivialization as the (manifold) direct product $J^{3} G=$ $G \times \mathcal{G} \times \mathcal{G} \times \mathcal{G}$ endowed with the group multiplication law ${ }^{3}$ and the inverse element

$$
\begin{gathered}
\left(g, x_{1}, x_{2}, x_{3}\right)\left(h, y_{1}, y_{2}, y_{3}\right)= \\
=\left(g h, x_{1}+{ }^{g} y_{1}, x_{2}+{ }^{g} y_{2}+\frac{1}{2}\left[x_{1},{ }^{g} y_{1}\right], x_{3}+{ }^{g} y_{3}+\frac{2}{3}\left[x_{1},{ }^{g} y_{2}\right]+\frac{1}{3}\left[x_{2},{ }^{g} y_{1}\right]+\frac{1}{6}\left[x_{1}\left[x_{1},{ }^{g} y_{1}\right]\right]\right), \\
\left(g, x_{1}, x_{2}, x_{3}\right)^{-1}=\left(g^{-1},-{ }^{g-1} x_{1},-{ }^{-1} x_{2},-{ }^{g-1} x_{3}+\frac{1}{3} g^{-1}\left[x_{1}, x_{2}\right]\right)
\end{gathered}
$$

where $g, h \in G, x_{i}, y_{i} \in \mathcal{G}, i=1,2,3$ and

$$
{ }^{g} y_{i}:=\operatorname{Ad}_{g} y_{i} .
$$

As the vector space, the Lie algebra $\mathcal{D}$ is the direct sum $\mathcal{D}=\mathcal{G} \oplus \mathcal{G} \oplus \mathcal{G} \oplus \mathcal{G}$ endowed with the Lie bracket

$$
\begin{gathered}
{\left[\left(\xi_{0}, \xi_{1}, \xi_{2}, \xi_{3}\right),\left(\chi_{0}, \chi_{1}, \chi_{2}, \chi_{3}\right)\right]=} \\
=\left(\left[\xi_{0}, \chi_{0}\right],\left[\xi_{0}, \chi_{1}\right]+\left[\xi_{1}, \chi_{0}\right],\left[\xi_{0}, \chi_{2}\right]+\left[\xi_{1}, \chi_{1}\right]+\left[\xi_{2}, \chi_{0}\right],\left[\xi_{0}, \chi_{3}\right]+\left[\xi_{1}, \chi_{2}\right]+\left[\xi_{2}, \chi_{1}\right]+\left[\xi_{3}, \chi_{0}\right]\right) .
\end{gathered}
$$

\footnotetext{
${ }^{2}$ The interpretation of the $3^{\text {rd }}$ order jet bundle of the semi-simple Lie group $G$ as the Drinfeld double comes from Ref. [37].

${ }^{3}$ The multiplication law $(5.58)$ differs from that given in $[37,52]$ by suitable normalization conventions, namely $x_{i} \in \mathcal{G}$ used in [52] are $i$ ! multiples of our $x_{i}$.
} 
Here, of course, $\xi_{i}, \chi_{i} \in \mathcal{G}, i=0,1,2,3$.

For convenience, we add the following useful formulas for the exponential map

$$
\exp \left(t\left(0, \xi_{1}, \xi_{2}, \xi_{3}\right)\right)=\left(e_{G}, t \xi_{1}, t \xi_{2}, t \xi_{3}+\frac{t^{2}}{6}\left[\xi_{1}, \xi_{2}\right]\right) \in J^{3} G
$$

and for the right-invariant Maurer-Cartan form on $J^{3} G$

$d l l^{-1}=\left(d g g^{-1}, \operatorname{Ad}_{g} d X_{1}, \operatorname{Ad}_{g}\left(d X_{2}+\frac{1}{2}\left[X_{1}, d X_{1}\right]\right), \operatorname{Ad}_{g}\left(d X_{3}+\left[X_{1}, d X_{2}\right]+\frac{1}{6}\left[X_{1},\left[X_{1}, d X_{1}\right]\right]\right)\right)$

where now we have parametrized the element $l=\left(g, x_{1}, x_{2}, x_{3}\right)$ differently, that is in terms of the products of "pure elements" (cf. [52])

$$
l=(g, 0,0,0)\left(e_{G}, X_{1}, 0,0\right)\left(e_{G}, 0, X_{2}, 0\right)\left(e_{G}, 0,0, X_{3}\right) .
$$

Note that the different parametrizations of the group $J^{3} G$ are related by the formulas:

$$
g=g, \quad x_{1}=\operatorname{Ad}_{g} X_{1}, \quad x_{2}=\operatorname{Ad}_{g} X_{2}, \quad x_{3}=\operatorname{Ad}_{g}\left(X_{3}+\frac{2}{3}\left[X_{1}, X_{2}\right]\right) .
$$

The split-signature symmetric non-degenerate ad-invariant bilinear form $(., .)_{\mathcal{D}}$ on the Lie algebra $\mathcal{D}$ is given by

$$
\left(\left(\xi_{0}, \xi_{1}, \xi_{2}, \xi_{3}\right),\left(\chi_{0}, \chi_{1}, \chi_{2}, \chi_{3}\right)\right)_{\mathcal{D}}=\left(\xi_{0}, \chi_{3}\right)_{\mathcal{G}}+\left(\xi_{1}, \chi_{2}\right)_{\mathcal{G}}+\left(\xi_{2}, \chi_{1}\right)_{\mathcal{G}}+\left(\xi_{3}, \chi_{0}\right)_{\mathcal{G}}
$$

Now we define an appropriate non-degenerate $\mathcal{E}$-model on the double $D$ by choosing the following operator $\mathcal{E}_{\mu}: \mathcal{D} \rightarrow \mathcal{D}$

$$
\mathcal{E}_{\mu}\left(\xi_{0}, \xi_{1}, \xi_{2}, \xi_{3}\right)=-\left(\mu^{3} \xi_{3}, \mu \xi_{2}, \mu^{-1} \xi_{1}, \mu^{-3} \xi_{0}\right)
$$

To obtain the degenerate $\hat{\mathcal{E}}$-model, we use the procedure of Section 3.1 and choose the isotropic subalgebra $\mathcal{F} \in \mathcal{D}$ as

$$
\mathcal{F}=\left\{\left(\xi_{0}, 0,0,0\right) \in D, \xi_{0} \in \mathcal{G}\right\}
$$

Consequently, the spaces $V_{ \pm}$are given by

$$
V_{ \pm}=\{(0, \mu \xi, \mp \xi, 0) \in \mathcal{D}, \xi \in \mathcal{G}\}
$$

We can associate the $\sigma$-model action (3.22) to the choices (5.68), (5.69) by choosing the maximally isotropic Lie subalgebra $\mathcal{K} \subset \mathcal{D}$ spanned by the elements of $\mathcal{D}$ of the form $\left(0,0, \xi_{2}, \xi_{3}\right)$. Fixing the gauge with respect to both $K$ and $F$ gauge symmetries as $l=\left(e_{G}, x, 0,0\right)$ we find

$$
d\left(e_{G}, x, 0,0\right)\left(e_{G}, x, 0,0\right)^{-1}=\left(0, d x, \frac{1}{2}[x, d x], \frac{1}{6}[x,[x, d x]]\right),
$$


therefore the WZ term in the action (3.22) is given by the expression

$$
\begin{gathered}
\frac{1}{4} \int \delta^{-1} \oint\left(\delta l l^{-1},\left[\partial_{\sigma} l l^{-1}, \delta l l^{-1}\right]\right)_{\mathcal{D}}= \\
=\frac{1}{4} \int \delta^{-1} \oint\left(\delta x,\left[\partial_{\sigma} x, \delta x\right]_{\mathcal{G}}\right)_{\mathcal{G}}=\frac{1}{6} \int d \tau \oint\left(x,\left[\partial_{\sigma} x, \partial_{\tau} x\right]_{\mathcal{G}}\right)_{\mathcal{G}} .
\end{gathered}
$$

Using the formula

$$
\operatorname{Ad}_{\left(e_{G}, x, 0,0\right)}\left(0,0, \xi_{2}, \xi_{3}\right)=\left(0,0, \xi_{2}, \xi_{3}+\left[x, \xi_{2}\right]\right)
$$

we obtain also

$$
W_{x}^{ \pm} \partial_{ \pm} l l^{-1}=W_{x}^{ \pm} \partial_{ \pm}\left(e_{G}, x, 0,0\right)\left(e_{G}, x, 0,0\right)^{-1}=\left(0, \partial_{ \pm} x, \mp \mu^{-1} \partial_{ \pm} x, 0\right),
$$

therefore the dressing coset action (3.22) finally reads

$$
S_{\mu}(x)=-\frac{1}{2 \mu} \int d \tau \oint\left(\left(\partial_{+} x, \partial_{-} x\right)_{\mathcal{G}}+\frac{\mu}{3}\left(x,\left[\partial_{+} x, \partial_{-} x\right]_{\mathcal{G}}\right)_{\mathcal{G}}\right) .
$$

We indeed recognize in the formula (5.75) the action of the pseudochiral model (5.57).

To prove the integrability of the pseudo-chiral model (5.75), we start with the ansatz

$$
\hat{O}(z)\left(0, \xi_{1}, \xi_{2}, 0\right)=p_{1}(z) \mu \xi_{1}+p_{2}(z) \mu^{2} \xi_{2}, \quad \xi_{1}, \xi_{2} \in \mathcal{G}
$$

where $p_{1}(z), p_{2}(z)$ are numerical functions that we look for. The condition (3.25) then gives

$$
p_{1}^{2}(z)-p_{2}^{2}(z)=p_{2}(z)
$$

which is solved by

$$
p_{1}(z)=\frac{-z}{1-z^{2}}, \quad p_{2}(z)=\frac{-1}{1-z^{2}}
$$

We find also easily the adjoint operator $O^{\dagger}(z): \mathcal{G} \rightarrow \mathcal{D}$

$$
O^{\dagger}(z) \xi=\left(0, p_{2}(z) \mu^{2} \xi, p_{1}(z) \mu \xi, \xi\right)
$$

Finally, the operator $\hat{r}(z, w): \mathcal{G} \rightarrow \mathcal{G}$ verifying the conditions (3.30) and (3.31) turns out to be also given by the multiplication by the numerical function

$$
\hat{r}(z, w) \xi=\frac{\mu^{3}}{1-w^{2}} \frac{\xi}{z-w} .
$$

In the present context, the polynomial $1-w^{2}$ is often referred to as the 'twist' function. 


\subsection{Generalized pseudo-chiral model with two fields}

Now we use our method to construct a genuinely new integrable dressing coset $\sigma$-model. For that we equip the $5^{\text {th }}$-order jet bundle $J^{5} G$ with the structure of the Drinfeld double. The group $D=J^{5} G$ can be conveniently parametrized via the right trivialization as the direct product of manifolds $J^{5} G=G \times \underbrace{\mathcal{G} \times \cdots \times \mathcal{G}}_{5 \text { times }}$ endowed with the group multiplication $\operatorname{law}^{4}$ and the inverse element

$$
\begin{gathered}
\left(g, x_{1}, x_{2}, x_{3}, x_{4}, x_{5}\right)\left(h, y_{1}, y_{2}, y_{3}, y_{4}, y_{5}\right)= \\
=\left(g h, x_{1}+{ }^{g} y_{1}, x_{2}+{ }^{g} y_{2}+\frac{1}{2}\left[x_{1},{ }^{g} y_{1}\right], x_{3}+{ }^{g} y_{3}+\frac{2}{3}\left[x_{1},{ }^{g} y_{2}\right]+\frac{1}{3}\left[x_{2},{ }^{g} y_{1}\right]+\frac{1}{6}\left[x_{1}\left[x_{1},{ }^{g} y_{1}\right]\right]\right. \\
x_{4}+{ }^{g} y_{4}+\frac{3}{4}\left[x_{1},{ }^{g} y_{3}\right]+\frac{1}{2}\left[x_{2},{ }^{g} y_{2}\right]+\frac{1}{4}\left[x_{3},{ }^{g} y_{1}\right]+\frac{1}{4}\left[x_{1}\left[x_{1},{ }^{g} y_{2}\right]\right]+\frac{1}{6}\left[x_{2}\left[x_{1},{ }^{g} y_{1}\right]\right]+\frac{1}{12}\left[x_{1}\left[x_{2},{ }^{g} y_{1}\right]\right]+ \\
+\frac{1}{24}\left[x_{1},\left[x_{1},\left[x_{1},{ }^{g} y_{1}\right]\right]\right], x_{5}+{ }^{g} y_{5}+\frac{4}{5}\left[x_{1},{ }^{g} y_{4}\right]+\frac{3}{5}\left[x_{2},{ }^{g} y_{3}\right]+\frac{2}{5}\left[x_{3},{ }^{g} y_{2}\right]+\frac{1}{5}\left[x_{4},{ }^{g} y_{1}\right]+ \\
+\frac{3}{10}\left[x_{1},\left[x_{1},{ }^{g} y_{3}\right]\right]+\frac{3}{20}\left[x_{3},\left[x_{1},{ }^{g} y_{1}\right]\right]+\frac{1}{20}\left[x_{1},\left[x_{3},{ }^{g} y_{1}\right]\right]+\frac{4}{15}\left[x_{2},\left[x_{1},{ }^{g} y_{2}\right]\right]+\frac{2}{15}\left[x_{1},\left[x_{2},{ }^{g} y_{2}\right]\right]+ \\
+\frac{1}{10}\left[x_{2},\left[x_{2},{ }^{g} y_{1}\right]\right]+\frac{1}{15}\left[x_{1},\left[x_{1},\left[x_{1},{ }^{g} y_{2}\right]\right]\right]+\frac{1}{20}\left[x_{2},\left[x_{1},\left[x_{1},{ }^{g} y_{1}\right]\right]\right]+\frac{1}{30}\left[x_{1},\left[x_{2},\left[x_{1},{ }^{g} y_{1}\right]\right]\right]+ \\
\left.+\frac{1}{60}\left[x_{1},\left[x_{1},\left[x_{2},{ }^{g} y_{1}\right]\right]\right]+\frac{1}{120}\left[x_{1},\left[x_{1},\left[x_{1},\left[x_{1},{ }^{g} y_{1}\right]\right]\right]\right]\right) \\
\quad\left(g, x_{1}, x_{2}, x_{3}, x_{4}, x_{5}\right)-1=\left(g^{-1},-{ }^{-1} x_{1},-{ }^{-1} x_{2},-{ }^{g} x_{3}+\frac{1}{3} g^{-1}\left[x_{1}, x_{2}\right],\right. \\
-\frac{1}{5} g^{-1} x_{4}+\frac{1}{2} g^{-1}\left[x_{2}, x_{3}\right]-\frac{3}{20} g^{-1}\left[x_{1},\left[x_{1}, x_{3}\right]\right]+\frac{1}{12} g^{-1}\left[x_{1},\left[x_{1}, x_{2}\right]\right],-{ }^{g}{ }^{-1} x_{5}+\frac{3}{5} g^{-1}\left[x_{2},\left[x_{2}, x_{1}\right]+\right. \\
\left.+\frac{1}{60} g^{-1}\left[x_{1},\left[x_{1},\left[x_{1}, x_{2}\right]\right]\right]\right)
\end{gathered}
$$

where $g, h \in G, x_{i}, y_{i} \in \mathcal{G}, i=1, \ldots, 5$ and

$$
{ }^{g} y_{i}:=\operatorname{Ad}_{g} y_{i}
$$

As the vector space, the Lie algebra $\mathcal{D}$ is the direct sum $\mathcal{D}=\underbrace{\mathcal{G} \oplus \cdots \oplus \mathcal{G}}_{6 \text { times }}$ endowed with the Lie bracket

$$
\begin{gathered}
{\left[\left(\xi_{0}, \xi_{1}, \ldots, \xi_{5}\right),\left(\chi_{0}, \chi_{1}, \ldots, \chi_{5}\right)\right]=\left(\left[\xi_{0}, \chi_{0}\right],\left[\xi_{0}, \chi_{1}\right]+\left[\xi_{1}, \chi_{0}\right],\left[\xi_{0}, \chi_{2}\right]+\left[\xi_{1}, \chi_{1}\right]+\left[\xi_{2}, \chi_{0}\right],\right.} \\
{\left[\xi_{0}, \chi_{3}\right]+\left[\xi_{1}, \chi_{2}\right]+\left[\xi_{2}, \chi_{1}\right]+\left[\xi_{3}, \chi_{0}\right],\left[\xi_{0}, \chi_{4}\right]+\left[\xi_{1}, \chi_{3}\right]+\left[\xi_{2}, \chi_{2}\right]+\left[\xi_{3}, \chi_{1}\right]+\left[\xi_{4}, \chi_{0}\right],} \\
\left.\left[\xi_{0}, \chi_{5}\right]+\left[\xi_{1}, \chi_{4}\right]+\left[\xi_{2}, \chi_{3}\right]+\left[\xi_{3}, \chi_{2}\right]+\left[\xi_{4}, \chi_{1}\right]+\left[\xi_{5}, \chi_{0}\right]\right) .
\end{gathered}
$$

Here, of course, $\xi_{i}, \chi_{i} \in \mathcal{G}, i=0,1, \ldots, 5$.

\footnotetext{
${ }^{4}$ The multiplication law $(5.81)$ differs from that given in $[37,52]$ by suitable normalization conventions, namely $x_{i} \in \mathcal{G}$ used in [52] are $i$ ! multiples of our $x_{i}$.
} 
For convenience, we add the following useful formulas for the exponential map $\exp \left(t\left(0, \ldots, 0, \xi_{j}, 0, \ldots, 0\right)\right)=\left(e_{G}, 0, \ldots, 0, t \xi_{j}, 0, \ldots, 0\right) \in J^{5} G, \quad j=1, \ldots, 5$

and for the right-invariant Maurer-Cartan form

$$
d l l^{-1}=d \tilde{g} \tilde{g}^{-1}+\operatorname{Ad}_{\tilde{g}} \sum_{j=1}^{5} \operatorname{Ad}_{\prod_{k=1}^{j-1} \tilde{X}_{k}} d \tilde{X}_{j} \tilde{X}_{j}^{-1},
$$

where we parametrized the group $J^{5} G$ in terms of the products of the "pure elements"

$$
\begin{gathered}
l=\tilde{g} \tilde{X}_{1} \tilde{X}_{2} \ldots \tilde{X}_{5} \equiv(g, 0,0,0,0,0)\left(e_{G}, X_{1}, 0,0,0,0\right) \times \\
\times\left(e_{G}, 0, X_{2}, 0,0,0\right)\left(e_{G}, 0,0, X_{3}, 0,0\right)\left(e_{G}, 0,0,0, X_{4}, 0\right)\left(e_{G}, 0,0,0,0, X_{5}\right) .
\end{gathered}
$$

Note also that

$$
\begin{gathered}
d \tilde{g} \tilde{g}^{-1}=\left(d g g^{-1}, 0,0,0,0,0\right), \\
d \tilde{X}_{1} \tilde{X}_{1}^{-1}=\left(0, d X_{1}, \frac{1}{2 !} \operatorname{ad}_{X_{1}} d X_{1}, \frac{1}{3 !} \operatorname{ad}_{X_{1}}^{2} d X_{1}, \frac{1}{4 !} \operatorname{ad}_{X_{1}}^{3} d X_{1}, \frac{1}{5 !} \operatorname{ad}_{X_{1}}^{4} d X_{1}\right), \\
d \tilde{X}_{2} \tilde{X}_{2}^{-1}=\left(0,0, d X_{2}, 0, \frac{1}{2 !} \operatorname{ad}_{X_{2}} d X_{2}, 0\right), \quad d \tilde{X}_{3} \tilde{X}_{3}^{-1}=\left(0,0,0, d X_{3}, 0,0\right), \\
d \tilde{X}_{4} \tilde{X}_{4}^{-1}=\left(0,0,0,0, d X_{4}, 0\right), \quad d \tilde{X}_{5} \tilde{X}_{5}^{-1}=\left(0,0,0,0,0, d X_{5}\right) .
\end{gathered}
$$

The split-signature symmetric non-degenerate ad-invariant bilinear form $(., .)_{\mathcal{D}}$ on the Lie algebra $\mathcal{D}$ is given by

$$
(\boldsymbol{\xi}, \boldsymbol{\chi})_{\mathcal{D}}=\left(\left(\xi_{0}, \xi_{1}, \ldots, \xi_{5}\right),\left(\chi_{0}, \chi_{1}, \ldots, \chi_{5}\right)\right)_{\mathcal{D}}=\sum_{j=0}^{5}\left(\xi_{j}, \chi_{5-j}\right)_{\mathcal{G}}
$$

Now we define an appropriate non-degenerate $\mathcal{E}$-model on the double $D$ by choosing the following operator $\mathcal{E}_{\mu}: \mathcal{D} \rightarrow \mathcal{D}$

$$
\mathcal{E}_{\mu}\left(\xi_{0}, \xi_{1}, \xi_{2}, \xi_{3}, \xi_{4}, \xi_{5}\right)=-\left(\mu^{5} \xi_{5}, \mu^{3} \xi_{4}, \mu \xi_{3}, \mu^{-1} \xi_{2}, \mu^{-3} \xi_{1}, \mu^{-5} \xi_{0}\right)
$$

To obtain the degenerate $\hat{\mathcal{E}}$-model, we use the procedure of Section 3.1 choose as the isotropic subalgebra $\mathcal{F} \in \mathcal{D}$ as

$$
\mathcal{F}=\left\{\left(\xi_{0}, 0,0,0,0,0\right) \in D, \xi_{0} \in \mathcal{G}\right\} .
$$

Consequently, the spaces $V_{ \pm}$are formed by the elements of the form

$$
\left(0, \mu^{3} \xi_{1}, \mu \xi_{2}, \mp \xi_{2}, \mp \xi_{1}, 0\right) \in V_{ \pm}
$$

We can associate the $\sigma$-model action (3.22) to the choices (5.92), (5.93) by choosing the maximally isotropic Lie subalgebra $\mathcal{K} \subset \mathcal{D}$ formed by the elements of the form

$$
\left(0,0,0, \xi_{3}, \xi_{4}, \xi_{5}\right) \in \mathcal{K}
$$


Fixing the gauge with respect to the both $K$ and $F$ as $l_{0}=\tilde{X}_{1} \tilde{X}_{2}$ we find from (5.85) and (5.90)

$$
\begin{gathered}
d\left(\tilde{X}_{1} \tilde{X}_{2}\right)\left(\tilde{X}_{1} \tilde{X}_{2}\right)^{-1}=\left(0, d X_{1}, d X_{2}+\frac{1}{2 !} \operatorname{ad}_{X_{1}} d X_{1}, \operatorname{ad}_{X_{1}} d X_{2}+\frac{1}{3 !} \operatorname{ad}_{X_{1}}^{2} d X_{1},\right. \\
\left.\frac{1}{2} \operatorname{ad}_{X_{2}} d X_{2}+\frac{1}{2} \operatorname{ad}_{X_{1}}^{2} d X_{2}+\frac{1}{4 !} \operatorname{ad}_{X_{1}}^{3} d X_{1}, \frac{1}{2} \operatorname{ad}_{X_{1}} \operatorname{ad}_{X_{2}} d X_{2}+\frac{1}{3 !} \operatorname{ad}_{X_{1}}^{3} d X_{2}+\frac{1}{5 !} \operatorname{ad}_{X_{1}}^{4} d X_{1}\right),
\end{gathered}
$$

therefore the WZ term in the action (3.22) is given by the expression

$$
\begin{gathered}
\frac{1}{4} \int \delta^{-1} \oint\left(\delta l_{0} l_{0}^{-1},\left[\partial_{\sigma} l_{0} l_{0}^{-1}, \delta l_{0} l_{0}^{-1}\right]\right)_{\mathcal{D}}=-\frac{1}{5 !} \int d \tau \oint\left(X_{1},\left[\operatorname{ad}_{X_{1}} \partial_{\sigma} X_{1}, \operatorname{ad}_{X_{1}} \partial_{\tau} X_{1}\right]_{\mathcal{G}}\right)_{\mathcal{G}}+ \\
+\frac{1}{4} \int d \tau \oint\left(X_{2},\left[\partial_{\sigma} X_{1}, \partial_{\tau} X_{2}\right]_{\mathcal{G}}+\left[\partial_{\sigma} X_{2}, \partial_{\tau} X_{1}\right]_{\mathcal{G}}\right)_{\mathcal{G}}+ \\
\frac{1}{12} \int d \tau \oint\left(\left(\operatorname{ad}_{X_{1}} \partial_{\sigma} X_{1}, \operatorname{ad}_{X_{1}} \partial_{\tau} X_{2}\right)_{\mathcal{G}}-\left(\operatorname{ad}_{X_{1}} \partial_{\sigma} X_{2}, \operatorname{ad}_{X_{1}} \partial_{\tau} X_{1}\right)_{\mathcal{G}}\right)
\end{gathered}
$$

Using the formula

$\operatorname{Ad}_{\tilde{X}_{1} \tilde{X}_{2}}\left(0,0,0, \xi_{3}, \xi_{4}, \xi_{5}\right)=\left(0,0,0, \xi_{3}, \xi_{4}+\left[X_{1}, \xi_{3}\right], \xi_{5}+\left[X_{1}, \xi_{4}\right]+\left[X_{2}, \xi_{3}\right]+\frac{1}{2}\left[X_{1},\left[X_{1}, \xi_{3}\right]\right]\right)$,

we obtain also

$$
\begin{gathered}
W_{X_{1}, X_{2}}^{ \pm} \partial_{ \pm} l_{0} l_{0}^{-1}=W_{X_{1}, X_{2}}^{ \pm} \partial_{ \pm}\left(\tilde{X}_{1} \tilde{X}_{2}\right)\left(\tilde{X}_{1} \tilde{X}_{2}\right)^{-1}= \\
=\left(0, \partial_{ \pm} X_{1}, \partial_{ \pm} X_{2}+\frac{1}{2} \operatorname{ad}_{X_{1}} \partial_{ \pm} X_{1}, \mp \mu^{-1}\left(\partial_{ \pm} X_{2}+\frac{1}{2} \operatorname{ad}_{X_{1}} \partial_{ \pm} X_{1}\right), \mp \mu^{-3} \partial_{ \pm} X_{1}, 0\right),
\end{gathered}
$$

therefore the dressing coset action (3.22) finally reads

$$
\begin{gathered}
S_{\mu}\left(X_{1}, X_{2}\right)= \\
-\frac{1}{2 \mu^{3}} \int d \tau \oint\left(\left(\partial_{+} X_{1}, \partial_{-} X_{1}\right)_{\mathcal{G}}+\mu^{2}\left(U_{+}, U_{-}\right)_{\mathcal{G}}+\mu^{3}\left(X_{1},\left[U_{+}, U_{-}\right]_{\mathcal{G}}\right)_{\mathcal{G}}\right)+ \\
\frac{1}{15} \int d \tau \oint\left(\operatorname{ad}_{X_{1}}^{3} \partial_{+} X_{1}, \partial_{-} X_{1}\right)_{\mathcal{G}}+\frac{1}{6} \int d \tau \oint\left(\left(\operatorname{ad}_{X_{1}}^{2} \partial_{+} X_{1}, U_{-}\right)_{\mathcal{G}}-\left(U_{+}, \operatorname{ad}_{X_{1}}^{2} \partial_{-} X_{1}\right)_{\mathcal{G}}\right)
\end{gathered}
$$

where

$$
U_{ \pm}:=\partial_{ \pm} X_{2}+\frac{1}{2} \operatorname{ad}_{X_{1}} \partial_{ \pm} X_{1}
$$

The action (5.100) is the 2-field generalization of the pseudo-chiral model of Zakharov et Mikhailov (5.75).

To prove the integrability of the model (5.100) by using the method developed in Section 3.2, we start with the ansatz

$$
\hat{O}(z)\left(0, \xi_{1}, \xi_{2}, \xi_{3}, \xi_{4}, 0\right)=\sum_{j=1}^{4} p_{j}(z) \mu^{j} \xi_{j} .
$$


The integrability condition (3.25) then require that the four unknown functions $p_{j}(z)$ are solutions of the following system of four equations

$$
\begin{gathered}
p_{1}^{2}(z)-p_{4}^{2}(z)=p_{2}(z), \quad p_{2}^{2}(z)-p_{3}^{2}(z)=p_{4}(z) \\
p_{1}(z) p_{2}(z)-p_{3}(z) p_{4}(z)=p_{3}(z), \quad p_{1}(z) p_{3}(z)-p_{2}(z) p_{4}(z)=p_{4}(z),
\end{gathered}
$$

or, equivalently,

$$
p_{i}(z) p_{j}(z)-p_{5-i}(z) p_{5-j}(z)=p_{i+j}(z), \quad 1 \leq i \leq j \leq i+j \leq 4 .
$$

The solution is

$$
\begin{gathered}
p_{1}(z)=\frac{z^{3}-2 z}{z^{4}-3 z^{2}+1}, \quad p_{2}(z)=\frac{z^{2}-1}{z^{4}-3 z^{2}+1}, \\
p_{3}(z)=\frac{z}{z^{4}-3 z^{2}+1}, \quad p_{4}(z)=\frac{1}{z^{4}-3 z^{2}+1},
\end{gathered}
$$

We find also easily the adjoint operator $O^{\dagger}(z): \mathcal{G} \rightarrow \mathcal{D}$

$$
O^{\dagger}(z) \xi=\left(0, p_{4}(z) \mu^{4} \xi, p_{3}(z) \mu^{3} \xi, p_{2}(z) \mu^{2} \xi, p_{1}(z) \mu \xi, \xi\right)
$$

Finally, we need the operator $\hat{r}(z, w): \mathcal{G} \rightarrow \mathcal{G}$ verifying the conditions (3.30) and (3.31). We assume that $\hat{r}(z, w)$ is given by the multiplication by a numerical function $\rho(z, w)$, that is

$$
\hat{r}(z, w) \xi=\mu^{5} \rho(z, w) \xi .
$$

This assumption works, because the conditions (3.30) and (3.31) become

$$
\sum_{j=0}^{m} p_{5-j}(z) p_{5+j-m}(w)+p_{5-m}(z) \rho(z, w)+p_{5-m}(w) \rho(w, z)=0
$$

where $m=1, \ldots, 5$ and we defined $p_{0}(z)=1$ and $p_{5}(z)=0$. The conditions (5.110) are then indeed solved by

$$
\rho(z, w)=\frac{-1}{w^{4}-3 w^{2}+1} \frac{1}{z-w} .
$$

In the present context, the polynomial $-\left(w^{4}-3 w^{2}+1\right)$ is often referred to as the 'twist' function.

\section{$5.3 n$-field generalization of the pseudochiral model}

Taking an integer $n$, we now equip the $(2 n+1)^{\text {th }}$-order jet bundle $J^{2 n+1} G$ with the structure of the Drinfeld double. The group $D=J^{2 n+1} G$ can be conveniently parametrized via the right trivialization as the direct product of manifolds 
$J^{2 n+1} G=G \times \underbrace{\mathcal{G} \times \cdots \times \mathcal{G}}_{(2 n+1) \text {-times }}$ endowed with the group multiplication law ${ }^{5}$ and the inverse element

$$
\begin{gathered}
\left(g, x_{1}, \ldots, x_{j}, \ldots\right)\left(h, y_{1}, \ldots y_{j}, \ldots\right)= \\
=\left(g h, x_{1}+\operatorname{Ad}_{g} y_{1}, \ldots, x_{j}+\sum_{i_{1}+\cdots+i_{l}=j} M_{i_{1} \ldots i_{l}} \operatorname{ad}_{x_{i_{l-1}}} \ldots \operatorname{ad}_{x_{i_{1}}} \operatorname{Ad}_{g} y_{i_{l}}, \ldots\right), \\
\left(g, x_{1}, \ldots, x_{j}, \ldots\right)^{-1}= \\
=\left(g^{-1},-\operatorname{Ad}_{g^{-1}} x_{1}, \ldots, \sum_{i_{1}+\cdots+i_{l}=j}(-1)^{l} M_{i_{1} \ldots i_{l}} \operatorname{Ad}_{g^{-1}} \operatorname{ad}_{x_{i_{1}}} \ldots \operatorname{ad}_{x_{i_{l-1}}} x_{i_{l}}, \ldots\right),
\end{gathered}
$$

where $g, h \in G, x_{i}, y_{i} \in \mathcal{G}, i=1, \ldots, 2 n+1$ and the numerical coefficients $M_{i_{1} \ldots i_{l}}$ are given by the formula

$$
M_{i_{1} \ldots i_{l}}=\prod_{k=1}^{l} \frac{i_{k}}{\sum_{m=1}^{k} i_{m}} \equiv \frac{i_{1}}{i_{1}} \times \frac{i_{2}}{i_{1}+i_{2}} \times \frac{i_{3}}{i_{1}+i_{2}+i_{3}} \times \cdots \times \frac{i_{l}}{i_{1}+\cdots+i_{l}} .
$$

Note also that $i_{m}$ are strictly positive integers and the sum $\sum_{i_{1}+\ldots i_{l}=j}$ runs over all possible partitions of the integer $j$. The order of the partition also matters, e.g. for the integer $j=3$ we have the partitions $3,2+1,1+2$ and $1+1+1$ or, in more detail, $i_{1}=3$, for $l=1, i_{1}=2, i_{2}=1$ as well as $i_{1}=1, i_{2}=2$ for $l=2$ and $i_{1}=i_{2}=i_{3}=1$ for $l=3$. The corresponding coefficients $M_{i_{1} \ldots i_{l}}$ read (cf. (5.58))

$$
M_{3}=1, \quad M_{12}=\frac{2}{3}, \quad M_{21}=\frac{1}{3}, \quad M_{111}=\frac{1}{6} .
$$

We remark in particular that $M_{12} \neq M_{21}$ which is another illustration of the fact that the order of the partition does matter.

As the vector space, the Lie algebra $\mathcal{D}$ is the direct sum $\mathcal{D}=\underbrace{\mathcal{G} \oplus \cdots \oplus \mathcal{G}}_{(2 n+2) \text {-times }}$ endowed with the Lie bracket

$$
[\boldsymbol{\xi}, \boldsymbol{\chi}]_{\mathcal{D}} \equiv\left[\left(\xi_{0}, \xi_{1}, \ldots, \xi_{2 n+1}\right),\left(\chi_{0}, \chi_{1}, \ldots, \chi_{2 n+1}\right)\right]_{\mathcal{D}}=\left([\boldsymbol{\xi}, \boldsymbol{\chi}]_{0},[\boldsymbol{\xi}, \boldsymbol{\chi}]_{1}, \ldots,[\boldsymbol{\xi}, \boldsymbol{\chi}]_{2 n+1}\right)
$$

where

$$
[\boldsymbol{\xi}, \boldsymbol{\chi}]_{m}:=\sum_{j=0}^{m}\left[\xi_{j}, \chi_{m-j}\right]_{\mathcal{G}}, \quad m=0, \ldots, 2 n+1 .
$$

Here, of course, $\boldsymbol{\xi}, \boldsymbol{\chi} \in \mathcal{D}, \xi_{i}, \chi_{i} \in \mathcal{G}, i=0,1, \ldots, 2 n+1$.

For convenience, we add the following useful formulas for the exponential map

$$
\exp \left(t\left(0, \ldots, 0, \xi_{j}, 0, \ldots, 0\right)\right)=\left(e_{G}, 0, \ldots, 0, t \xi_{j}, 0, \ldots, 0\right) \in J^{2 n+1} G
$$

\footnotetext{
${ }^{5}$ The multiplication law (5.58) differs from that given in $[37,52]$ by suitable normalization conventions, namely $x_{i} \in \mathcal{G}$ used in [52] are $i$ ! multiples of our $x_{i}$.
} 
and for the right-invariant Maurer-Cartan form

$$
d l l^{-1}=d \tilde{g} \tilde{g}^{-1}+\operatorname{Ad}_{\tilde{g}} \sum_{j=1}^{2 n+1} \operatorname{Ad}_{\prod_{k=1}^{j-1} \tilde{X}_{k}} d \tilde{X}_{j} \tilde{X}_{j}^{-1}
$$

where we parametrized the group $J^{2 n+1} G$ in terms of the products of the "pure elements"

$$
\begin{gathered}
l=\tilde{g} \tilde{X}_{1} \tilde{X}_{2} \ldots \tilde{X}_{2 n+1} \equiv \\
\equiv(g, 0, \ldots, 0)\left(e_{G}, X_{1}, 0, \ldots, 0\right)\left(e_{G}, 0, X_{2}, 0, \ldots, 0\right) \ldots\left(e_{G}, 0, \ldots, 0, X_{2 n+1}\right) .
\end{gathered}
$$

Note also that

$$
\begin{gathered}
d \tilde{g} \tilde{g}^{-1}=\left(d g g^{-1}, 0, \ldots, 0\right), \\
d \tilde{X}_{j} \tilde{X}_{j}^{-1}=(0, \ldots, 0, d X_{j}, 0, \ldots, 0, \underbrace{\frac{1}{2}\left[X_{j}, d X_{j}\right]}_{(2 j)^{\text {th }} \text { place }}, 0, \ldots, \underbrace{\frac{1}{n !} \operatorname{ad}_{X_{j}}^{n-1} d X_{j}}_{(n j)^{\text {th }} \text { place }}, 0, \ldots) .
\end{gathered}
$$

The split-signature symmetric non-degenerate ad-invariant bilinear form $(., .)_{\mathcal{D}}$ on the Lie algebra $\mathcal{D}$ is given by

$$
(\boldsymbol{\xi}, \boldsymbol{\chi})_{\mathcal{D}}=\left(\left(\xi_{0}, \xi_{1}, \ldots, \xi_{2 n+1}\right),\left(\chi_{0}, \chi_{1}, \ldots, \chi_{2 n+1}\right)\right)_{\mathcal{D}}=\sum_{j=0}^{2 n+1}\left(\xi_{j}, \chi_{2 n+1-j}\right)_{\mathcal{G}}
$$

Now we define an appropriate non-degenerate $\mathcal{E}$-model on the double $D$ by choosing the following operator $\mathcal{E}_{\mu}: \mathcal{D} \rightarrow \mathcal{D}$

$$
\begin{gathered}
\mathcal{E}_{\mu}\left(\xi_{0}, \xi_{1}, \ldots, \xi_{2 n}, \xi_{2 n+1}\right)= \\
=-\left(\mu^{2 n+1} \xi_{2 n+1}, \mu^{2 n-1} \xi_{2 n}, \ldots, \mu^{3} \xi_{n+2}, \mu \xi_{n+1}, \mu^{-1} \xi_{n}, \mu^{-3} \xi_{n-1}, \ldots, \mu^{-(2 n-1)} \xi_{1}, \mu^{-(2 n+1)} \xi_{0}\right) .
\end{gathered}
$$

To obtain the degenerate $\hat{\mathcal{E}}$-model, we use the procedure of Section 3.1 and choose the isotropic subalgebra $\mathcal{F} \in \mathcal{D}$ as

$$
\mathcal{F}=\left\{\left(\xi_{0}, 0, \ldots, 0\right) \in \mathcal{D}, \xi_{0} \in \mathcal{G}\right\} .
$$

Consequently, the spaces $V_{ \pm}$are given by

$$
V_{ \pm}=\left(0, \mu^{2 n-1} \xi_{1}, \ldots, \mu^{3} \xi_{n-1}, \mu \xi_{n}, \mp \xi_{n}, \mp \xi_{n-1}, \ldots, \mp \xi_{1}, 0\right) .
$$

We can associate the $\sigma$-model action $(3.22)$ to the choices $(5.124),(5.125)$ by choosing the maximally isotropic Lie subalgebra $\mathcal{K} \subset \mathcal{D}$ defined as

$$
\mathcal{K}=\left\{\boldsymbol{\xi} \in D, \xi_{j}=0, j \leq n\right\} .
$$

that is $\mathcal{K}$ is spanned by the elements of $\mathcal{D}$ of the form $\left(0, \ldots, 0, \xi_{n+1}, \ldots, \xi_{2 n+1}\right)$. Fixing the gauge with respect to the both $K$ and $F$ as $l_{0}=\tilde{X}_{1} \tilde{X}_{2} \ldots \tilde{X}_{n}$ we find

$$
d l_{0} l_{0}^{-1}=\sum_{j=1}^{n} \operatorname{Ad}_{\prod_{k=1}^{j-1} \tilde{X}_{k}} d \tilde{X}_{j} \tilde{X}_{j}^{-1}
$$


therefore the WZ term in the action (3.22) is given by the expression

$$
\begin{gathered}
\frac{1}{4} \int \delta^{-1} \oint\left(\delta l_{0} l_{0}^{-1},\left[\partial_{\sigma} l_{0} l_{0}^{-1}, \delta l_{0} l_{0}^{-1}\right]_{\mathcal{D}}\right)_{\mathcal{D}}= \\
\frac{1}{4} \int \delta^{-1} \oint \sum_{k=1}^{2 n} \sum_{j=0}^{2 n+1-k}\left(\left(\delta l_{0} l_{0}^{-1}\right)_{k},\left[\left(\partial_{\sigma} l_{0} l_{0}^{-1}\right)_{j},\left(\delta l_{0} l_{0}^{-1}\right)_{2 n+1-k-j}\right]_{\mathcal{G}}\right)_{\mathcal{G}}
\end{gathered}
$$

where $\left(\delta l_{0} l_{0}^{-1}\right)_{j} \in \mathcal{G}$ stand for the $j^{\text {th }}$ component of the Maurer-Cartan form $\delta l_{0} l_{0}^{-1} \in \mathcal{D}$. Furthermore, using the formula

$$
\operatorname{Ad}_{\tilde{X}_{1} \ldots \tilde{X}_{n}} \mathcal{K}=\mathcal{K}
$$

we obtain also

$$
\begin{gathered}
W_{X}^{ \pm} \partial_{ \pm} l_{0} l_{0}^{-1}= \\
\left(0,\left(\partial_{ \pm} l_{0} l_{0}^{-1}\right)_{1}, \ldots,\left(\partial_{ \pm} l_{0} l_{0}^{-1}\right)_{n}, \mp \mu^{-1}\left(\partial_{ \pm} l_{0} l_{0}^{-1}\right)_{n}, \ldots, \mp \mu^{-(2 n-1)}\left(\partial_{ \pm} l_{0} l_{0}^{-1}\right)_{1}, 0\right) .
\end{gathered}
$$

Inserting the formulas (5.131) and (5.129) into the second order dressing coset action (3.22) we obtain the $n$-field generalization of the pseudochiral model

$$
\begin{gathered}
S_{\mu}\left(X_{1}, \ldots, X_{n}\right)=-\frac{1}{2} \int d \tau \oint \sum_{j=1}^{n} \mu^{-(2 n+1-2 j)}\left(\left(\partial_{+} l_{0} l_{0}^{-1}\right)_{j},\left(\partial_{-} l_{0} l_{0}^{-1}\right)_{j}\right)_{\mathcal{G}}+ \\
+\frac{1}{4} \int d \tau \oint \sum_{j=1}^{n}\left(\left(\left(\partial_{+} l_{0} l_{0}^{-1}\right)_{j},\left(\partial_{-} l_{0} l_{0}^{-1}\right)_{2 n+1-j}\right)_{\mathcal{G}}-\left(\left(\partial_{+} l_{0} l_{0}^{-1}\right)_{2 n+1-j},\left(\partial_{-} l_{0} l_{0}^{-1}\right)_{j}\right)_{\mathcal{G}}\right)_{+} \\
+\frac{1}{4} \int \delta^{-1} \oint \sum_{k=1}^{2 n} \sum_{j=0}^{2 n+1-k}\left(\left(\delta l_{0} l_{0}^{-1}\right)_{k},\left[\left(\partial_{\sigma} l_{0} l_{0}^{-1}\right)_{j},\left(\delta l_{0} l_{0}^{-1}\right)_{2 n+1-k-j}\right]_{\mathcal{G}}\right)_{\mathcal{G}}
\end{gathered}
$$

We remark that the action (5.132) of the $n$-field pseudochiral model employs just the components of the Maurer-Cartan form, which means in fact that it is written in the coordinate-invariant way (the first line correspond to the metric part while the second and third one to the Kalb-Ramond part). Of course, we could express it in the coordinates $X_{j}$ given by the formulas (5.120) by calculating correspondingly the components of the Maurer-Cartan form from (5.128). However, the result of such calculation is quite cumbersome and, anyway, not very illuminating as it can be observed by looking at the 2-field formula (5.100), where we did work out this procedure in detail.

To prove the integrability of the model (5.132), we start with the ansatz

$$
\hat{O}(z)\left(0, \xi_{1}, \xi_{2}, \ldots, \xi_{2 n-1}, \xi_{2 n}, 0\right)=\sum_{j=1}^{2 n} p_{j}(z) \mu^{j} \xi_{j} .
$$


The (weak) integrability condition (3.25) then require that the $2 n$ unknown functions $p_{j}(z)$ are solutions of the following system of $n^{2}$ equations

$$
p_{i}(z) p_{j}(z)-p_{2 n+1-i}(z) p_{2 n+1-j}(z)=p_{i+j}(z), \quad 1 \leq i \leq j \leq i+j \leq 2 n .
$$

The system is overdetermined for $n>2$, nevertheless it does possess the needed one-parameter family of solutions. Actually, we find those solutions quite easily taking inspiration from the quadratic identities holding for the Fibonacci numbers. The result is

$$
p_{2 k}(z)=(-1)^{k} \frac{\cos (n-k+1 / 2) \theta}{\cos (n+1 / 2) \theta}, p_{2 k-1}(z)=(-1)^{k} \frac{\sin (n-k+1) \theta}{\cos (n+1 / 2) \theta},
$$

where $k=1, \ldots, n$ and

$$
z=2 \sin \frac{\theta}{2}
$$

We find also easily the adjoint operator $O^{\dagger}(z): \mathcal{G} \rightarrow \mathcal{D}$

$$
O^{\dagger}(z) \xi=\left(0, p_{2 n}(z) \mu^{2 n} \xi, p_{2 n-1}(z) \mu^{2 n-1} \xi, \ldots, p_{2}(z) \mu^{2} \xi, p_{1}(z) \mu \xi, \xi\right) .
$$

Finally, we need the operator $\hat{r}(z, w): \mathcal{G} \rightarrow \mathcal{G}$ verifying the (strong) integrability conditions (3.30) and (3.31). We assume that $\hat{r}(z, w)$ is given by the multiplication by a numerical function $\rho(z, w)$, that is

$$
\hat{r}(z, w) \xi=\mu^{2 n+1} \rho(z, w) \xi .
$$

This assumption works, because the conditions (3.30) and (3.31) become

$$
\sum_{j=0}^{m} p_{2 n+1-j}(z) p_{2 n+1+j-m}(w)+p_{2 n+1-m}(z) \rho(z, w)+p_{2 n+1-m}(w) \rho(w, z)=0,
$$

where $m=0, \ldots, 2 n+1$ and we defined $p_{0}(z)=1$ and $p_{2 n+1}(z)=0$. The conditions (5.139) are then indeed solved by

$$
\rho(z, w)=\frac{(-1)^{n} \cos \frac{\psi}{2}}{2 \cos (2 n+1) \frac{\psi}{2}} \times \frac{1}{\sin \frac{\psi}{2}-\sin \frac{\theta}{2}},
$$

where

$$
z=2 \sin \frac{\theta}{2}, \quad w=2 \sin \frac{\psi}{2}
$$

In the present context, the 'twist' function is $(-1)^{n+1} \frac{\cos (2 n+1) \frac{\psi}{2}}{\cos \frac{\psi}{2}}$ which is the polynomial function if expressed in the variable $w$.

Remark 5.1. To verify, that the function $\rho(z, w)$ given by the expression (5.140) indeed solves the conditions (5.139), it is useful to use the identity

$$
f(\phi)+f(\phi+\alpha)+f(\phi+2 \alpha)+\cdots+f(\phi+n \alpha)=\frac{\sin (n+1) \frac{\alpha}{2}}{\sin \frac{\alpha}{2}} f\left(\phi+\frac{n \alpha}{2}\right),
$$

where $f$ stands either for sin or for cos. 


\section{Conclusions and outlook}

In this paper, we formulated the sufficient conditions for the strong integrability of the dressing cosets. We have also reformulated the non-deformed, $\lambda$-deformed and $\eta$-deformed symmetric space cosets as the dressing cosets and we have shown that the integrability of those three theories can be established by solving our sufficient conditions. Finally, we have introduced the new class of dressing cosets based on higher order jet bundles of quadratic Lie groups playing the role of the Drinfeld doubles. Those new theories can be interpreted as the (interacting) $n$ field generalizations of the pseudo-chiral $\sigma$-model of Zakharov and Mikhailov. We have solved our sufficient conditions of the strong integrability also in this case and identified the so called twist functions of those theories which turn out to have poles only in the infinity.

As far as the outlook is concerned, we find appealing to study in future how our results could be put in the perspective of two other frameworks which are currently used along with the $\mathcal{E}$-models to study integrable $\sigma$-models on group manifolds, namely, the formalism based on the $4 \mathrm{~d}$ Chern-Simons gauge theory [8] as well as the one based on the structure of the affine Gaudin models [11,51].

\section{References}

[1] D. Bykov, Complex structure-induced deformations of $\sigma$-models, JHEP 03 (2017) 130, arXiv:1611.07116 [hep-th]

[2] J. Balog, P. Forgács, Z. Horváth and L. Palla, A new family of SU(2) symmetric integrable $\sigma$-models, Phys. Lett. B324 (1994) 403, hep-th/9307030

[3] O. Babelon, D. Bernard and M.Talon, Introduction to classical integrable systems, Cambridge University Press, Cambridge (2003)

[4] O. Babelon, C.M. Viallet, Hamiltonian structures and Lax equations, Phys. Lett. B237 (1989), 411

[5] R. Borsato, A.A. Tseytlin and L. Wulff, Supergravity background of $\lambda$ deformed model for AdS2 × S2 supercoset, Nucl.Phys. B905 (2016) 264-292, arXiv:1601.08192 [hep-th]

[6] A. Cabrera and H. Montani, Hamiltonian loop group actions and T-duality for group manifolds, J.Geom.Phys. 56 (2006) 1116-1143, hep-th/0412289

[7] I. V. Cherednik; Relativistically invariant quasiclassical limits of integrable two-dimensional quantum models, Theor. Math. Phys. 47 (1981) 422

[8] K. Costello and M. Yamazaki, Gauge Theory And Integrability, III, arXiv:1908.02289 [hep-th] 
[9] F. Delduc, M. Magro and B. Vicedo, Alleviating the non-ultralocality of coset sigma models through a generalized Faddeev-Reshetikhin procedure, JHEP 08 (2012) 019, arXiv:1204.0766 [hep-th].

[10] F. Delduc, M. Magro and B. Vicedo, On classical q-deformations of integrable sigma-models, JHEP 11 (2013), 192, arXiv:1308.3581 [hep-th].

[11] F. Delduc, S. Lacroix, M. Magro and B. Vicedo, Assembling integrable $\sigma$ models as affine Gaudin models, JHEP 06 (2019), 017, arXiv:1903.00368 [hepth]

[12] S. Demulder, S. Driezen, A. Sevrin and D. C. Thompson, Classical and Quantum Aspects of Yang-Baxter Wess-Zumino Models, JHEP 03 (2018), 041, arXiv:1711.00084 [hep-th]

[13] S. Demulder, F. Hassler, G. Piccinini and D. C. Thompson, Generalised Cosets, JHEP 09 (2020), 044, arXiv:1912.11036 [hep-th]

[14] S. Demulder, F. Hassler, G. Piccinini and D. C. Thompson, Integrable deformation of $\mathbb{C P}^{n}$ and generalised Kähler geometry, JHEP 10 (2020) 086, arXiv:2002.11144 [hep-th]

[15] V.A. Fateev, The sigma model (dual) representation for a two-parameter family of integrable quantum field theories, Nucl. Phys. B473 (1996) 509

[16] F. Delduc, B. Hoare, T. Kameyama and M. Magro, Combining the bi-YangBaxter deformation, the Wess-Zumino term and TsT transformations in one integrable $\sigma$-model, JHEP 10 (2017) 212, arXiv:1707.08371 [hep-th]

[17] G. Georgiou, K. Sfetsos and K. Siampos, Strong integrability of $\lambda$-deformed models, Nucl. Phys. B 952 (2020), 114923, arXiv:1911.07859 [hep-th].

[18] F. Hassler, D. Lüst and F. J. Rudolph, Para-Hermitian geometries for Poisson-Lie symmetric $\sigma$-models, JHEP 10 (2019), 160, arXiv:1905.03791 [hep-th]

[19] B. Hoare and S. Lacroix, Yang-Baxter deformations of the principal chiral model plus Wess-Zumino term, J. Phys. A 53 (2020) no.50, 505401, arXiv:2009.00341 [hep-th]

[20] B. Hoare and F. Seibold, Poisson-Lie duals of the $\eta$-deformed AdS2×S2×T6 superstring, JHEP 1808 (2018) 107, arXiv:1807.04608 [hep-th]

[21] B. Hoare and A.A. Tseytlin, On integrable deformations of superstring sigma models related to $A d S_{n} \times S^{n}$ supercosets, Nucl. Phys. B897 (2015) 448, arXiv:1504.07213[hep-th]

[22] T. J. Hollowood, J. L. Miramontes and D. M. Schmidtt, S-Matrices and Quantum Group Symmetry of k-Deformed Sigma Models, J. Phys. A 49 (2016) no.46, 465201, arXiv:1506.06601 [hep-th] 
[23] I. Kawaguchi, T. Matsumoto and K. Yoshida, Jordanian deformations of the $A d S_{5} \times S^{5}$ superstring JHEP 04 (2014) 153, arXiv:1401.4855 [hep-th]

[24] C. Klimčík, Yang-Baxter $\sigma$-model and dS/AdS T-duality, JHEP 0212 (2002) 051, hep-th/0210095

[25] C. Klimčík, Integrability of the Yang-Baxter $\sigma$-model, J. Math. Phys. 50 (2009) 043508, arXiv:0802.3518 [hep-th]

[26] C. Klimčík, Integrability of the bi-Yang-Baxter $\sigma$-model, Lett. Math. Phys. 104 (2014) 1095, arXiv:1402.2105 [math-ph]

[27] C. Klimčík, $\eta$ and $\lambda$ deformations as $\mathcal{E}$-models, Nucl. Phys. B900 (2015) 259, arXiv:1508.05832 [hep-th]

[28] C. Klimčík, Poisson-Lie T-duals of the bi-Yang-Baxter models, Phys. Lett. B 760 (2016), 345-349, arXiv:1606.03016 [hep-th]

[29] C. Klimčík, Yang-Baxter $\sigma$-model with WZNW term as $\mathcal{E}$-model, Phys.Lett. B772 (2017) 725-730, arXiv:1706.08912 [hep-th]

[30] C. Klimčík, Dressing cosets and multi-parametric integrable deformations, JHEP 1907 (2019) 176, arXiv:1903.00439 [hep-th]

[31] C. Klimčík, Strong integrability of the bi- YB-WZ model, Lett. Math. Phys. 110 (2020), 2397-2416, arXiv:2001.05466 [hep-th]

[32] C. Klimčík, Brief lectures on duality, integrability and deformations, Rev. Math. Phys. 33 (2021) no.06, 2130004, arXiv:2101.05230 [hep-th]

[33] C. Klimčík and P. Ševera, Dual non-Abelian duality and the Drinfeld double, Phys. Lett. B351 (1995) 455-462, hep-th/9502122; C. Klimčík, Poisson-Lie T-duality, Nucl. Phys. (Proc. Suppl.) B46 (1996) 116-121, hep-th/9509095; P. Ševera, Minimálne plochy a dualita, Diploma thesis, Prague University, 1995, in Slovak

[34] C. Klimčík and P. Ševera, Poisson-Lie T-duality and loop groups of Drinfeld doubles, Phys. Lett. B372 (1996), 65-71, hep-th/9512040

[35] C. Klimčík and P. Ševera, Dressing cosets, Phys. Lett. B381 (1996) 56-61, hep-th/9602162

[36] C. Klimčík and P. Ševera, Non-Abelian momentum-winding exchange, Phys.Lett. B383 (1996) 281-286, hep-th/9605212

[37] S. Lacroix and B. Vicedo, Integrable $\mathcal{E}$-models, 4d Chern-Simons theory and affine Gaudin models, I - Lagrangian aspects, SIGMA 17 (2021), 058, arXiv:2011.13809 [hep-th] 
[38] P. D. Lax, Integrals of non linear equations of evolution and solitary waves, Comm. Pure Appl. Math. 21, (1968), 467.16

[39] J.-H. Lu and A. Weinstein, Poisson Lie groups, dressing transformations, and Bruhat decompositions, J. Diff. Geom. 31 (1990) 510

[40] J. M. Maillet, Kac Moody algebra and extended Yang Baxter relations in the O(n) nonlinear sigma model, Phys. Lett. B162 (1985), 137

[41] N. Mohammedi, On some integrable deformations of the Wess-Zumino-Witten model, arXiv:2012.09753 [hep-th]

[42] D. Osten and S. J. van Tongeren, Abelian Yang-Baxter Deformations and TsT transformations, Nucl. Phys. B915 184 (2017), arXiv:1608.08504 [hep-th]

[43] T. B. Rochais, Geometric Approaches to Quantum Fields and Strings at Strong Couplings, arXiv:2105.07067 [hep-th]

[44] P. Ševera, On integrability of 2-dimensional $\sigma$-models of Poisson-Lie type, JHEP 11 (2017), 015 arXiv:1709.02213 [hep-th]

[45] P. Ševera and F. Valach, Courant algebroids, Poisson-Lie T-duality, and type II supergravities, arXiv:1810.07763 [math.DG]

[46] K. Sfetsos, Duality invariant class of two-dimensional field theories Nucl. Phys. B561, (1999) 316, hep-th/9904188; Poisson-Lie T duality beyond the classical level and the renormalization group, Phys.Lett. B432 (1998) 365-375, hep-th/9803019

[47] K. Sfetsos, Integrable interpolations: From exact CFTs to non-Abelian Tduals, Nucl. Phys. B 880 (2014) 225, arXiv:1312.4560 [hep-th]

[48] K. Sfetsos, K. Siampos and D. Thompson, Generalised integrable $\lambda$ and $\eta$-deformations and their relation, Nucl.Phys. B899 (2015) 489-512, arXiv:1506.05784 [hep-th]

[49] A. Stern, T duality for coset models, Nucl.Phys. B557 (1999) 459-479, hepth/9903170

[50] E. K. Sklyanin, On complete integrability of the Landau Lifschitz equation, Zap. Nauch. Sem. LOMI95(1980), 55

[51] B. Vicedo, On integrable field theories as dihedral affine Gaudin models, Int. Math. Res. Not. 2020 (2020) no.15, 4513-4601, arXiv:1701.04856 [hep-th]

[52] C. Vizman, The group structure for jet bundles over Lie groups, J. Lie Theory 23 (2013), 885-897, arXiv:1304.5024 [math] 
[53] V. Zakharov and A. Mikhailov, Relativistically invariant two-dimensional models of field theory which are integrable by means of the inverse scattering problem method, Zh. Eksp. Teor. Fiz. 74, 1953 (1978) [Sov. Phys. JETP 47 (1978) 1017] 